\title{
Accessing baryon to meson transition distribution amplitudes in meson production in association with a high invariant mass lepton pair at GSI-FAIR with PANDA
}

\author{
J. P. Lansberg ${ }^{1}$, B. Pire ${ }^{2}$, K. Semenov-Tian-Shansky ${ }^{3}$, L. Szymanowski ${ }^{4}$ \\ ${ }^{1}$ IPNO, Université Paris-Sud, CNRS/IN2P3, 91406 Orsay, France \\ ${ }^{2}$ CPhT, École Polytechnique, CNRS, 91128 Palaiseau, France \\ ${ }^{3}$ IFPA, département AGO, Université de Liège, 4000 Liège, Belgium \\ ${ }^{4}$ National Center for Nuclear Research (NCBJ), 00-681 Warsaw, Poland
}

\begin{abstract}
Nucleon-antinucleon annihilation into a near backward- (or forward-) produced meson and a high invariant mass lepton pair admits a factorized description in terms of antinucleon (or nucleon) distribution amplitudes (DAs) and nucleon to meson (or antinucleon to meson) transition distribution amplitudes (TDAs). We estimate the cross section of backward (and forward) pion and $\eta$-meson production in association with a high invariant mass lepton pair for the kinematical conditions of GSI-FAIR. The cross sections are found to be large enough to be measured with the PANDA detector. Interesting phenomenological applications of the approach are thus expected.

PACS numbers: 13.60.-r, 13.60.Le, 14.20.Dh
\end{abstract}




\section{INTRODUCTION}

The ambitious experimental program for the PANDA facility at GSI-FAIR (see 【1, 2]) will provide the experimental access to new classes of hard reactions for which the factorized description applies. Study of these reactions will open a new eyehole to look on hadron's interior and will bring a new perspective to the problem of hadronic structure in terms of the fundamental degrees of freedom of QCD.

A tempting possibility is to consider nucleon-antinucleon annihilation into a lepton pair with a high invariant mass $q^{2} \equiv Q^{2}$ in association with a light meson $\mathcal{M}=\{\pi, \eta, \rho, \omega, \ldots\}$ :

$$
\bar{N}\left(p_{\bar{N}}\right) N\left(p_{N}\right) \rightarrow \gamma^{*}(q) \mathcal{M}\left(p_{\mathcal{M}}\right) \rightarrow \ell^{+}\left(p_{\ell^{+}}\right) \ell^{-}\left(p_{\ell^{-}}\right) \mathcal{M}\left(p_{\mathcal{M}}\right)
$$

The analysis of [3], 4] , identifies two similar factorization regimes for the reaction (1) corresponding to forward and backward peaks of the meson production cross section.

Let us now specify our conventions ${ }^{1}$. The $z$-axis is chosen along the colliding nucleonantinucleon. For the PANDA setup it is natural to select as the positive direction of $z$ the direction the antinucleon is moving in the $\bar{N} N$ center-of-mass (CMS) frame.

- Within these conventions, the kinematical regime in which the $u$-channel momentum transfer squared, $u \equiv\left(p_{N}-p_{\mathcal{M}}\right)^{2}$, is small corresponds to the meson moving in the direction of the initial nucleon that is the backward direction.

- Analogously, the kinematical regime in which the $t$-channel momentum transfer squared, $t \equiv\left(p_{\bar{N}}-p_{\mathcal{M}}\right)^{2}$, is small, corresponds to the meson moving in the direction of the initial antinucleon that is the forward direction.

A detailed account of the corresponding kinematics is presented in Appendix A.

The backward and forward factorization regimes for the reaction (11) are casted with the help of two collinear factorization theorems schematically depicted on Fig. 1.

1. For backward kinematics the collinear factorization theorem (see the left panel of Fig. 1) is valid once $s=\left(p_{N}+p_{\bar{N}}\right)^{2} \equiv W^{2}$ and the invariant mass of the lepton pair $q^{2} \equiv Q^{2}$ are large; the corresponding skewness variable $\xi^{u}$ (A2), which characterizes

\footnotetext{
${ }^{1}$ Definitions of $t$ and $u$ here match Ref. [5] and differ from those of Ref. [4]
} 
the $u$-channel longitudinal momentum transfer, is fixed; $|u|$ is small as compared to $Q^{2}$ and $W^{2}$. The meson $\mathcal{M}$ is produced in the near backward direction in the $\bar{N} N$ CMS. We refer to this kinematics as the backward, or the $u$-channel, factorization regime.

2. Analogously, the t-channel collinear factorization theorem presented on the right panel of Fig. 1 is valid once $W^{2}$ and $Q^{2}$ are large; the corresponding skewness variable $\xi^{t}(\underline{\mathrm{A} 11})$ is fixed; $|t|$ is small as compared to $Q^{2}$ and $W^{2}$. The meson $\mathcal{M}$ is produced in the near forward direction in $\bar{N} N \mathrm{CMS}$, and we refer to this kinematics as the forward, or the $t$-channel, factorization regime.

It is worth emphasizing that for the moment the rigorous proof of the collinear factorization theorems for meson production in association with a high invariant mass lepton pair in $N \bar{N}$ annihilation as well as for backward meson electroproduction does not exist. Some physical arguments in favor of this kind of factorization for the spacelike version of the reaction (7) were presented in Refs. [6, 7]. In this paper we do not aim to prove the relevant factorization theorems but rather perform the feasibility estimates to try to see some evidences of the factorized description in the experiment.

The factorization theorems for the reaction (11) presented in Fig. 1 involve two kinds of nonperturbative objects: the conventional antibaryon (or baryon) distribution amplitudes (DAs) and the baryon to meson (or antibaryon to meson) transition distribution amplitudes (TDAs). The baryon to meson (antibaryon to meson) TDAs are defined through baryon (antibaryon)-meson matrix elements of the nonlocal three quark (antiquark) operator on the light cone [8 -12$]$ :

$$
\begin{aligned}
& \hat{O}_{\rho \tau \chi}^{\alpha \beta \gamma}\left(\lambda_{1} n, \lambda_{2} n, \lambda_{3} n\right)=\varepsilon_{c_{1} c_{2} c_{3}} \Psi_{\rho}^{c_{1} \alpha}\left(\lambda_{1} n\right) \Psi_{\tau}^{c_{2} \beta}\left(\lambda_{2} n\right) \Psi_{\chi}^{c_{3} \gamma}\left(\lambda_{3} n\right) \\
& \hat{\bar{O}}_{\alpha \beta \gamma \rho \tau \chi}\left(\lambda_{1} n, \lambda_{2} n, \lambda_{3} n\right)=\varepsilon_{c_{1} c_{2} c_{3}} \bar{\Psi}_{\alpha \rho}^{c_{1}}\left(\lambda_{1} n\right) \bar{\Psi}_{\beta \tau}^{c_{2}}\left(\lambda_{2} n\right) \bar{\Psi}_{\gamma \chi}^{c_{3}}\left(\lambda_{3} n\right) .
\end{aligned}
$$

Here $\alpha, \beta, \gamma$ stand for quark (antiquark) flavor indices and $\rho, \tau, \chi$ denote the Dirac spinor indices. Antisymmetrization stands over the color group indices $c_{1,2,3}{ }^{2}$. The gauge links in (2) can be omitted in the light-like gauge $A^{+}=0$. For the case when the meson $\mathcal{M}$ is a pseudoscalar, to the leading twist-3 accuracy, the form factor decomposition of $\mathcal{M N}$

\footnotetext{
${ }^{2}$ See App. B1 for the details of our index conventions.
} 

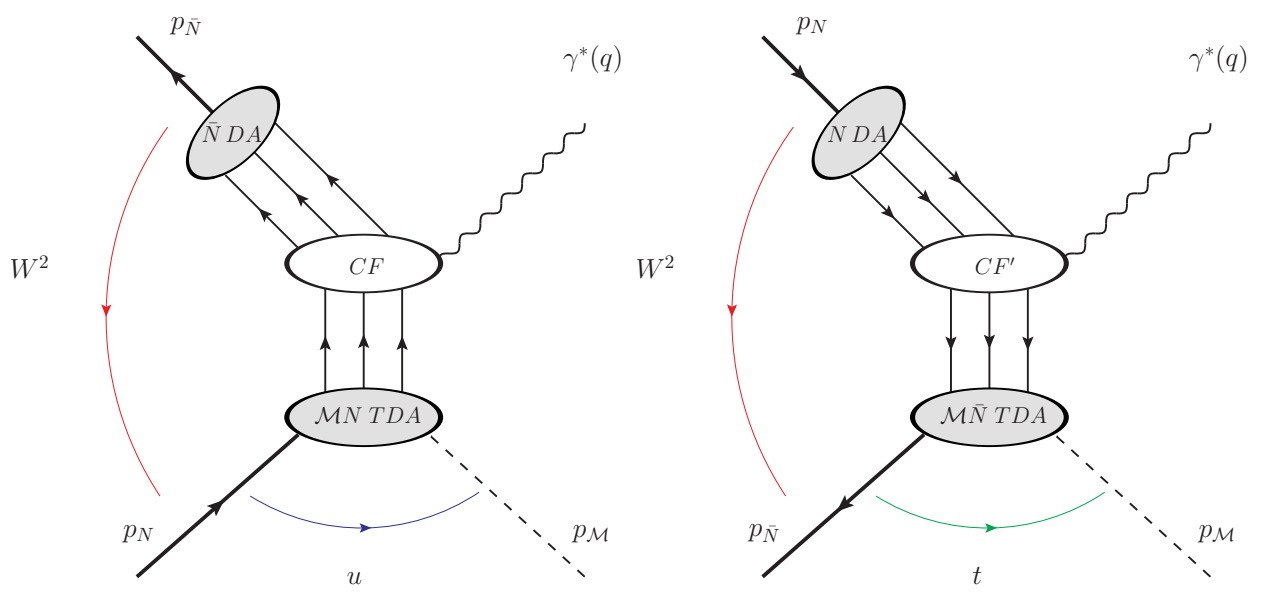

FIG. 1: Two possibilities for collinear factorization of the annihilation process $N \bar{N} \rightarrow \gamma^{*}(q) \mathcal{M}\left(p_{\mathcal{M}}\right)$. Left panel: backward kinematics $(|u| \sim 0)$. Right panel: forward kinematics $(|t| \sim 0) . \bar{N}(N)$ DA stands for the distribution amplitude of antinucleon (nucleon); $\mathcal{M} N(\mathcal{M} \bar{N})$ TDA stands for the transition distribution amplitude from a nucleon (antinucleon) to a meson; $\mathrm{CF}$ and $\mathrm{CF}^{\prime}$ denote hard subprocess amplitudes (coefficient functions).

$(\mathcal{M} \bar{N})$ matrix elements of (2) involves eight invariant $\mathcal{M} N(\mathcal{M} \bar{N}) \operatorname{TDAs} H^{\mathcal{M} N}\left(H^{\mathcal{M}} \bar{N}\right)$, each being a function of the three longitudinal momentum fractions $x_{i}\left(\sum_{i} x_{i}=2 \xi\right)$, of the corresponding skewness variable $\xi$ and of the momentum transfer squared $\Delta^{2}$,

$$
\begin{aligned}
& H^{(\mathcal{M N}),(\mathcal{M N})}\left(x_{1}, x_{2}, x_{3}, \xi^{u, t}, \Delta^{u, t^{2}}\right) \\
& \equiv\left\{V_{1,2}^{(\mathcal{M N}),(\mathcal{M N})}, A_{1,2}^{(M N),(M \bar{N})}, T_{1,2,3,4}^{(\mathcal{M N}),(\mathcal{M} \bar{N})}\right\}\left(x_{1}, x_{2}, x_{3}, \xi^{u, t}, \Delta^{u, t^{2}}\right) .
\end{aligned}
$$

The isotopic symmetry and the charge conjugation invariance further reduce the number of independent nucleon to meson TDAs. In particular, $C$-invariance allows us to express all $\mathcal{M} \bar{N}$ TDAs through $\mathcal{M} N$ TDAs (see App. B).

Extensive studies of the properties and of the physical interpretation of $\pi N$ TDAs are presented in Refs. [3 [5, 13-15]. Conceptually, the $\mathcal{M N}$ TDAs share many common features both with the GPDs and the nucleon DAs. Indeed, the crossing transformation relates $\mathcal{M} N$ TDAs with the $\overline{\mathcal{M}} N$ generalized distribution amplitudes (GDAs), defined as the matrix element of the same light cone operator between $\overline{\mathcal{M}} N$ state and the vacuum. In the pion case, this allows to establish useful constraints from the chiral dynamics since $\pi N$ GDAs reduce to combinations of the usual nucleon DAs in the soft pion limit. In 
addition, $\pi N$ TDAs were recently estimated within the light-cone quark model [16]. The hadronic matrix elements of QCD operators are extensively studied on the lattice. In particular, $\pi N$ matrix elements of the operators corresponding to the Mellin moments of $\pi N$ TDAs have been recently considered on the lattice by several groups, see [17], [18] and Refs. therein.

On the other hand, similarly to the GPD case [19 22], a comprehensible physical picture may be obtained by Fourier transforming $\mathcal{M N}$ TDAs to the impact parameter space.

\section{MODELLING $\pi N$ TDAS}

First estimates of the cross section of $\pi^{0}$ production in association with a high- $Q^{2}$ lepton pair in $\bar{p} p$ annihilation for PANDA@GSI-FAIR conditions within the factorized description involving $\pi N$ TDAs were presented in [4]. In this analysis, an oversimplified model for $\pi N$ TDAs based on the soft pion theorem was employed. This model has obvious drawbacks: its domain of applicability is limited to the immediate vicinity of the soft-pion threshold; TDAs in this model do not have any intrinsic $\Delta_{T}^{2}$ dependence and finally, the cross channel leading baryon exchange contribution, which turns out to be dominant in many cases, was not taken into account.

In Ref. [5], a two component model for $\pi N$ TDAs was proposed. It includes the spectral part casted in terms of quadruple distributions and a $D$-term like contribution which is determined by the nucleon pole exchange in the cross channel (see Fig. 2). Quadruple distributions are fixed with the help of the soft pion theorem for $\pi N$ TDAs in terms of nucleon DAs. Once the pion mass is neglected $(m=0)$ in the strict soft pion limit $(\xi=1$, $\Delta^{2}=M^{2}$ ), this model gives the same predictions for the $N \bar{N} \rightarrow \ell^{+} \ell^{-} \pi$ cross section as the model employed in [4]. 

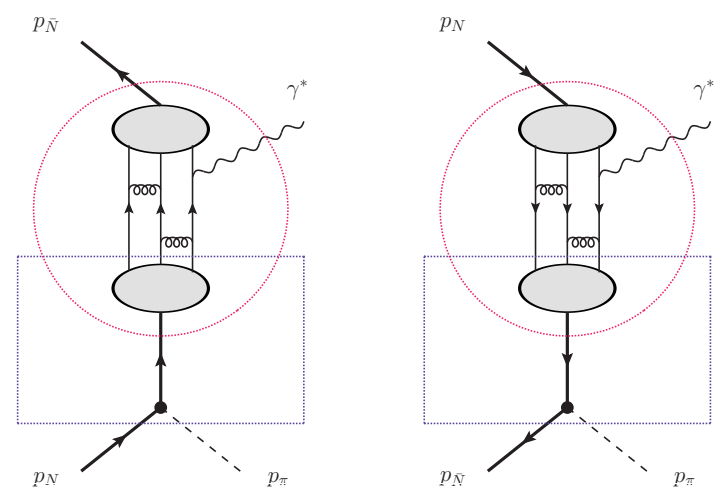

FIG. 2: Nucleon pole exchange model for $\pi N$ (left) and $\pi \bar{N}$ (right) TDAs; dashed circles contain typical LO graph for the nucleon (antinucleon) electromagnetic form factor in perturbative QCD; rectangles contain the nucleon pole contribution into $\pi N(\pi \bar{N})$ TDAs.

As it was found out in [5], the contribution of the spectral part dominates the cross section for large values of $\xi \approx 1$ (which is difficult to reach in experiments), while for small and intermediate values of $\xi$, the nucleon pole exchange part provides the dominant contribution to the $N \bar{N} \rightarrow \gamma^{*} \pi$ cross section.

In Sec. 41, we present our estimates of $N \bar{N} \rightarrow \ell^{+} \ell^{-} \pi$ cross section within our factorization scheme in the $u$-channel factorization regime ${ }^{3}$ (near backward pion production) using the nucleon pole exchange model for $\pi N$ TDAs.

The explicit expression for the contribution of the cross-channel nucleon exchange into the isospin- $\frac{1}{2} \pi N$ TDAs was obtained in [15],

$$
\begin{aligned}
& \left.\left\{V_{1}, A_{1}, T_{1}\right\}^{\left(\pi^{0} p\right)}\left(x_{i}, \xi, \Delta^{2}\right)\right|_{N(940)} \\
& =\Theta_{\operatorname{ERBL}}\left(x_{1}, x_{2}, x_{3}\right) \times\left(g_{\pi N N}\right) \frac{M f_{\pi}}{\Delta^{2}-M^{2}} \frac{1}{(2 \xi)}\left\{V^{p}, A^{p}, T^{p}\right\}\left(\frac{x_{1}}{2 \xi}, \frac{x_{2}}{2 \xi}, \frac{x_{3}}{2 \xi}\right) \\
& \left.\left\{V_{2}, A_{2}, T_{2}\right\}^{\left(\pi^{0} p\right)}\left(x_{i}, \xi, \Delta^{2}\right)\right|_{N(940)}=\left.\frac{1}{2}\left\{V_{1}, A_{1}, T_{1}\right\}^{\left(\pi^{0} p\right)}\left(x_{i}, \xi, \Delta^{2}\right)\right|_{N(940)} \\
& \left.\left\{T_{3}, T_{4}\right\}^{\left(\pi^{0} p\right)}\left(x_{i}, \xi, \Delta^{2}\right)\right|_{N(940)}=0,
\end{aligned}
$$

where $V^{p}, A^{p}$ and $T^{p}$ stand for the nucleon DAs; $g_{\pi N N} \approx 13$ is the pion-nucleon phe-

${ }^{3}$ In the remainder of the paper, in most cases we omit superscripts $u$ and $t$ for the kinematical quantities $\left(\xi, \Delta^{2}, p, n\right.$ etc. $)$ referring to the factorization regime. 
nomenological coupling and

$$
\Theta_{\mathrm{ERBL}}\left(x_{1}, x_{2}, x_{3}\right) \equiv \prod_{k=1}^{3} \theta\left(0 \leq x_{k} \leq 2 \xi\right)
$$

ensures the pure Efremov-Radyushkin-Brodsky-Lepage (ERBL) support.

For $\pi^{-} n$ TDAs, we get

$$
\begin{aligned}
& \left.\left\{V_{1,2}, A_{1,2}, T_{1,2}\right\}^{\left(\pi^{-} n\right)}\left(x_{i}, \xi, \Delta^{2}\right)\right|_{N(940)}=\left.\sqrt{2}\left\{V_{1,2}, A_{1,2}, T_{1,2}\right\}^{\left(\pi^{0} p\right)}\left(x_{i}, \xi, \Delta^{2}\right)\right|_{N(940)} ; \\
& \left.\left\{T_{3}, T_{4}\right\}^{\left(\pi^{-} n\right)}\left(x_{i}, \xi, \Delta^{2}\right)\right|_{N(940)}=0 .
\end{aligned}
$$

We also present some estimates of the $N \bar{N} \rightarrow \ell^{+} \ell^{-} \pi$ cross section within the two component model for $\pi N$ TDAs of Ref. [5].

\section{CROSS SECTION OF $\bar{N} N \rightarrow \gamma^{*} \pi \rightarrow \pi \ell^{+} \ell^{-}$}

Below we review the formulas for the cross section of

$$
N\left(p_{N}, s_{N}\right)+\bar{N}\left(p_{\bar{N}}, s_{\bar{N}}\right) \rightarrow \gamma^{*}(q)+\pi\left(p_{\pi}\right) \rightarrow \ell^{+}\left(p_{\ell^{+}}\right)+\ell^{-}\left(p_{\ell^{-}}\right)+\pi\left(p_{\pi}\right)
$$

established in [4]. The starting point is the general formula for the unpolarized differential cross section of the reaction (77) (see e.g. [23]),

$$
d \sigma=\frac{1}{2(2 \pi)^{5} \Lambda\left(W^{2}, M^{2}, M^{2}\right)}\left|\overline{\mathcal{M}^{N \bar{N} \rightarrow \ell^{+} \ell^{-} \pi}}\right|^{2} d_{3}(\mathrm{LIPS})
$$

where $\Lambda(x, y, z)=\sqrt{x^{2}+y^{2}+z^{2}-2 x y-2 x z-2 y z}$ is the usual Mandelstam function.

Following the standard procedure, the 3-particle differential Lorentz invariant phase space $d_{3}$ (LIPS) is decomposed into two 2-particle phase subspaces: those of $\gamma^{*} \pi$ and $\ell^{+} \ell^{-}$ systems. The former can be easily computed in $\bar{N} N$ CMS while the latter is computed in $\ell^{+} \ell^{-}$CMS yielding the result,

$$
d \sigma=\frac{1}{2(2 \pi)^{5} \Lambda\left(W^{2}, M^{2}, M^{2}\right)}\left|\overline{\mathcal{M}^{N \bar{N} \rightarrow \ell^{+} \ell^{-} \pi}}\right|^{2} \frac{d \Omega_{\pi}^{*}}{8 W^{2}} \Lambda\left(W^{2}, Q^{2}, m^{2}\right) \frac{d \Omega_{\ell}}{8},
$$

where $d \Omega_{\pi}^{*} \equiv d \cos \theta_{\pi}^{*} d \varphi_{\pi}^{*}$ in $N \bar{N}$ CMS. By $d \Omega_{\ell} \equiv d \cos \theta_{\ell} d \varphi_{\ell}$, we denote the produced lepton solid angle in $\ell^{+} \ell^{-}$CMS. By expressing $\cos \theta_{\pi}^{*}$ through $u=\left(p_{N}-p_{\pi}\right)^{2}$,

$$
d u=\frac{d \cos \theta_{\pi}^{*}}{2 W^{2}} \Lambda\left(W^{2}, M^{2}, M^{2}\right) \Lambda\left(W^{2}, Q^{2}, m^{2}\right)
$$


and integrating over the azimuthal angle $\varphi_{\pi}^{*}$ of the produced pion and over the azimuthal angle of the lepton pair $\varphi_{\ell}$, the following formula for the differential cross section of the reaction (7) is established:

$$
\frac{d \sigma}{d u d Q^{2} d \cos \theta_{\ell}}=\frac{\int d \varphi_{\ell}\left|\overline{\mathcal{M}^{N \bar{N} \rightarrow \ell^{+} \ell^{-}}}\right|^{2}}{64 W^{2}\left(W^{2}-4 M^{2}\right)(2 \pi)^{4}}
$$

The next step is to express the average-squared amplitude $\left|\overline{\mathcal{M}^{N \bar{N} \rightarrow \ell^{+} \ell^{-} \pi}}\right|^{2}$ through the amplitude $\mathcal{M}_{\lambda}^{s_{N} s_{\bar{N}}}$ of $N \bar{N} \rightarrow \gamma^{*} \pi$. Within the factorized approach of [4], at leading order in $\alpha_{s}$, the amplitude $\mathcal{M}_{\lambda}^{s_{N} s_{\bar{N}}}$ reads

$$
\mathcal{M}_{\lambda}^{s_{N} s_{\bar{N}}}=\mathcal{C} \frac{1}{Q^{4}}\left[\mathcal{S}_{\lambda}^{s_{N} s_{\bar{N}}} \mathcal{I}\left(\xi, \Delta^{2}\right)-\mathcal{S}_{\lambda}^{s_{N} s_{\bar{N}}} \mathcal{I}^{\prime}\left(\xi, \Delta^{2}\right)\right]
$$

where

$$
\mathcal{C}=-i \frac{\left(4 \pi \alpha_{s}\right)^{2} \sqrt{4 \pi \alpha_{e m}} f_{N}^{2}}{54 f_{\pi}} .
$$

Here, $f_{\pi}=93 \mathrm{MeV}$ is the pion weak decay constant and $f_{N}$ is a constant, which determines the value of the dimensional nucleon wave function at the origin [12]; $\alpha_{e m} \simeq \frac{1}{137}$ is the electromagnetic fine structure constant and $\alpha_{s} \simeq \frac{1}{3}$ is the strong coupling constant.

The spin structures $\mathcal{S}$ and $\mathcal{S}^{\prime}$ are defined as

$$
\begin{aligned}
& \mathcal{S}_{\lambda}^{s_{N} s_{\bar{N}}} \equiv \bar{V}\left(p_{\bar{N}}, s_{\bar{N}}\right) \hat{\epsilon}^{*}(\lambda) \gamma_{5} U\left(p_{N}, s_{N}\right) \\
& {\mathcal{S}_{\lambda}^{\prime s_{N} s_{\bar{N}}}}_{{ }_{\lambda}} \equiv \frac{1}{M} \bar{V}\left(p_{\bar{N}}, s_{\bar{N}}\right) \hat{\epsilon}^{*}(\lambda) \hat{\Delta}_{T} \gamma_{5} U\left(p_{N}, s_{N}\right) .
\end{aligned}
$$

We employ the standard "hat" notation for the contraction of a four-vector with the Dirac matrices. $\epsilon(\lambda)$ stands for the polarization vector of the virtual photon. $\mathcal{I}$ and $\mathcal{I}^{\prime}$ denote the convolution integrals of $\pi N$ TDAs and antinucleon DAs with the hard scattering kernels computed from the set of 21 relevant scattering diagrams (see [13]):

$$
\begin{aligned}
& \left\{\mathcal{I}, \mathcal{I}^{\prime}\right\}\left(\xi, \Delta^{2}\right) \equiv \int_{-1+\xi}^{1+\xi} d x_{1} \int_{-1+\xi}^{1+\xi} d x_{2} \int_{-1+\xi}^{1+\xi} d x_{3} \delta\left(x_{1}+x_{2}+x_{3}-2 \xi\right) \\
& \times \int_{0}^{1} d y_{1} \int_{0}^{1} d y_{2} \int_{0}^{1} d y_{3} \delta\left(y_{1}+y_{2}+y_{3}-1\right)\left(2 \sum_{\alpha=1}^{7}\left\{R_{\alpha}, R_{\alpha}^{\prime}\right\}+\sum_{\alpha=8}^{14}\left\{R_{\alpha}, R_{\alpha}^{\prime}\right\}\right) .
\end{aligned}
$$

The integrals in $x_{i}$ 's $\left(y_{i}\right.$ 's) stand over the support of $\pi N$ TDA (antinucleon DA).

For the $u$-channel factorization regime of $p \bar{p} \rightarrow \pi^{0} \ell^{+} \ell^{-}$, the coefficients $R_{\alpha}$ and $R_{\alpha}^{\prime}$ $(\alpha=1, . ., 14)$ correspond to the coefficients $T_{\alpha}$ and $T_{\alpha}^{\prime}$ presented in Table I of [13] up 
to an overall irrelevant phase factor $\eta_{N}^{*}{ }^{-1} \eta_{q}^{-3}$ and to the replacement $-i \varepsilon \rightarrow i \varepsilon$ in the denominators of hard scattering kernels. This latter change mirrors the fact that we consider $\gamma^{*}$ in the final state rather than in the initial state as it is for backward pion electroproduction.

We also note that Ref. [13] employed a somewhat inadequate parametrization of $\pi N$ TDAs, which does not maintain the polynomiality property in its simple form. In this paper, we use the parametrization of [15] which maintains the property of polynomiality. The relation between the two parametrizations is presented in App. A of [5]. For $n \bar{p} \rightarrow$ $\pi^{-} \ell^{+} \ell^{-}$reaction the coefficients $R_{\alpha}$ and $R_{\alpha}^{\prime}$ are the same as for $p \bar{p} \rightarrow \pi^{0} \ell^{+} \ell^{-}$channel up to an obvious change

$$
\left\{V_{1,2}, A_{1,2}, T_{1,2,3,4}\right\}^{\left(\pi^{0} p\right)} \rightarrow\left\{V_{1,2}, A_{1,2}, T_{1,2,3,4}\right\}^{\left(\pi^{-} n\right)}
$$

The differential cross section for unpolarized beam and unpolarized target is computed from the squared amplitude (12) averaged over spins of the initial particles:

$$
\left|\overline{\mathcal{M}_{\lambda \lambda^{\prime}}}\right|^{2}=\frac{1}{4} \sum_{s_{N} s_{\bar{N}}} \mathcal{M}_{\lambda}^{s_{N} s_{\bar{N}}}\left(\mathcal{M}_{\lambda^{\prime}}^{s_{N} s_{\bar{N}}}\right)^{*}
$$

with the given combination of photon helicities $\lambda, \lambda^{\prime}=T, L .\left|\overline{\mathcal{M}_{L L}}\right|^{2}\left|\overline{\mathcal{M}_{L T}}\right|^{2}$ and $\left|\overline{\mathcal{M}_{T L}}\right|^{2}$ vanish at the leading twist accuracy. We employ the relation

$$
\sum_{\lambda_{T}} \epsilon^{\nu}(\lambda) \epsilon^{\mu *}(\lambda)=-g^{\mu \nu}+\frac{1}{(p \cdot n)}\left(p^{\mu} n^{\nu}+p^{\nu} n^{\mu}\right)
$$

to sum over the transverse polarizations of the virtual photon and we get

$$
\left|\overline{\mathcal{M}_{T}}\right|^{2} \equiv \sum_{\lambda_{T}}\left|\overline{\mathcal{M}_{T T}}\right|^{2}=\frac{1}{4}|\mathcal{C}|^{2} \frac{2(1+\xi)}{\xi Q^{6}}\left(|\mathcal{I}|^{2}-\frac{\Delta_{T}^{2}}{M^{2}}\left|\mathcal{I}^{\prime}\right|^{2}\right)
$$

where $\mathcal{C}$ is defined in (13).

The averaged-squared amplitude for the process (7) reads

$$
\left|\overline{\mathcal{M}^{N \bar{N} \rightarrow \ell^{+} \ell^{-} \pi}}\right|^{2}=\frac{1}{4} \sum_{s_{p}, s_{\bar{p}}, \lambda, \lambda^{\prime}} \mathcal{M}_{\lambda}^{s_{p} s_{\bar{p}}} \frac{1}{Q^{2}} e^{2} \operatorname{Tr}\left\{\hat{p}_{\ell^{-}} \hat{\epsilon}(\lambda) \hat{p}_{\ell^{+}} \hat{\epsilon}^{*}\left(\lambda^{\prime}\right)\right\} \frac{1}{Q^{2}}\left(\mathcal{M}_{\lambda^{\prime}}^{s_{p} s_{\bar{p}}}\right)^{*}
$$

As pointed above, at the leading twist accuracy, only the transverse polarization of the virtual photon is contributing. After computing the relevant trace in (20) in the $\ell^{+} \ell^{-}$ CMS and integrating over the lepton polar angle $\varphi_{\ell}$, we finally get:

$$
\left.\int d \varphi_{\ell}\left|\overline{\mathcal{M}^{N \bar{N} \rightarrow \ell^{+} \ell^{-} \pi}}\right|^{2}\right|_{\text {Leading twist }}=\left|\overline{\mathcal{M}_{T}}\right|^{2} \frac{2 \pi e^{2}\left(1+\cos ^{2} \theta_{\ell}\right)}{Q^{2}} .
$$


A kinematical cut in $\Delta_{T}^{2}$, that is equivalent to a cut in $\cos \theta_{\pi}^{*}$ (A7), is a convenient way to select near backward and near forward kinematical configurations. On Fig. [3 with solid lines, we show the dependence of the CMS scattering angles $\theta_{\pi}^{*}$ (see eqs. (A7), (A16)) for the $u$-channel (left panel) and the $t$-channel (right panel) factorization regimes as the function of $\Delta_{T \min }^{u 2}$ and $\Delta_{T \min }^{t^{2}}$ respectively, for $W^{2}=5 \mathrm{GeV}^{2}, \xi=0.3$. For these kinematical parameters, the $\Delta_{T \min }^{u 2}=-0.2 \mathrm{GeV}^{2}$ cut corresponds to selecting pions flying backward into a cone with the aperture of $\sim 120^{\circ}$ in the $\bar{N} N$ CMS.

In order to implement the effect of the $\Delta_{T}^{2}$ cut, we consider the cross section integrated over the appropriate bin in $u$. Indeed, $u$ can be expressed as follows as the function of $\xi$ and $\Delta_{T}^{2}$ :

$$
u=\frac{\Delta_{T}^{2}(\xi+1)}{1-\xi}+\frac{2 \xi\left(M^{2}(1-\xi)-m^{2}(\xi+1)\right)}{1-\xi^{2}} .
$$

Therefore, the bin $\Delta_{T \min }^{2} \leq \Delta_{T}^{2} \leq 0$ corresponds to the following bin in $u$ : $u_{\min } \leq u \leq$ $u_{\max }$, where

$$
\begin{aligned}
& u_{\min }\left(\xi, \Delta_{T \min }^{2}\right)=\frac{\Delta_{T \min }^{2}(\xi+1)}{1-\xi}+\frac{2 \xi\left(M^{2}(1-\xi)-m^{2}(\xi+1)\right)}{1-\xi^{2}} \\
& u_{\max }(\xi)=\frac{2 \xi\left(M^{2}(1-\xi)-m^{2}(\xi+1)\right)}{1-\xi^{2}}
\end{aligned}
$$



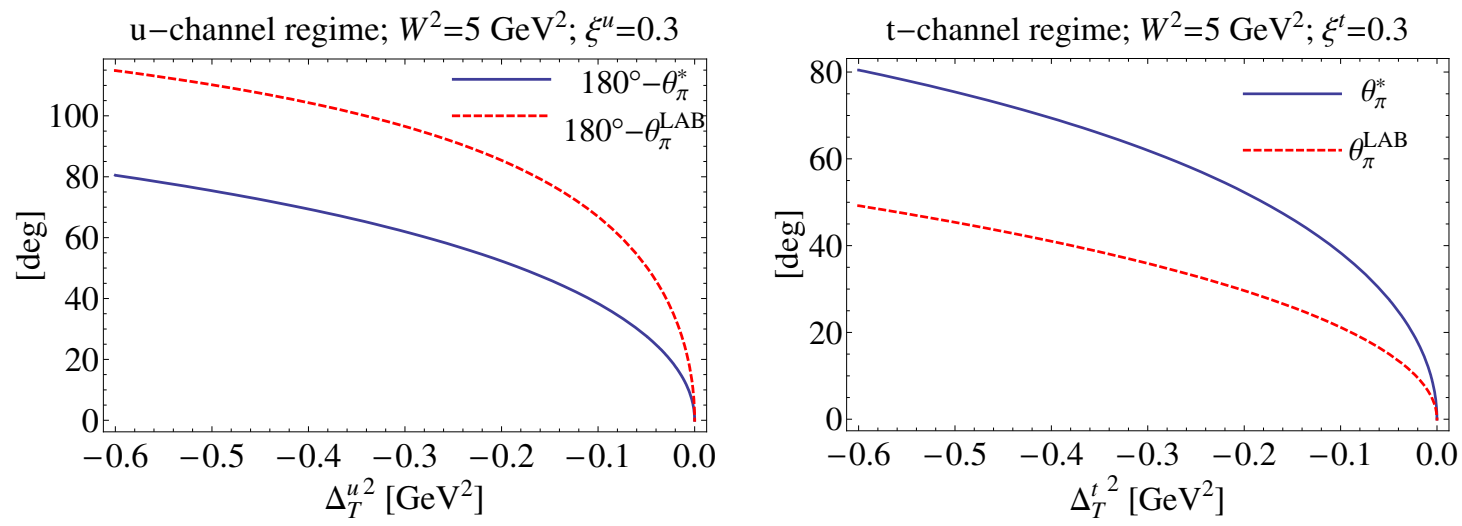

FIG. 3: The solid lines illustrate the dependence of the CMS scattering angles $\theta_{\pi}^{*}$ for the $u$-channel (left panel) and the $t$-channel (right panel) factorization regimes for the reaction (17) as functions of $\Delta_{T \min }^{u^{2}}$ and $\Delta_{T \min }^{t^{2}}$, respectively. The dashed lines illustrate the dependence of the LAB frame scattering angles $\theta_{\pi}^{\mathrm{LAB}}$ for the two factorization regimes as the function of $\Delta_{T \min }^{u 2}$ and $\Delta_{T \text { min }}^{t^{2}}$, respectively. Note that the forward peak is narrowed and the backward peak is broadened due to the effect of the boost from the CMS to the LAB frame which corresponds to the nucleon $N$ at rest in the $\overline{\mathrm{P}} \mathrm{ANDA}$ setup.

\section{PSEUDOSCALAR MESON PRODUCTION IN ASSOCIATION WITH A HIGH INVARIANT MASS LEPTON PAIR IN $\bar{N} N$ ANNIHILATION}

\section{Results for $\pi^{0}$ and $\pi^{-}$production}

On Figs. 4 and 5, we show our model predictions for the integrated cross section

$$
\frac{d \bar{\sigma}}{d Q^{2}}\left(\Delta_{T \min }^{2}\right) \equiv \int_{u_{\min }}^{u_{\max }} d u \int d \theta_{\ell} \frac{d \sigma}{d u d Q^{2} d \cos \theta_{\ell}}
$$

of $\bar{p} p \rightarrow \ell^{+} \ell^{-} \pi^{0}$ and of of $\bar{p} n \rightarrow \ell^{+} \ell^{-} \pi^{-}$as the function of $Q^{2}$, for several values of $W^{2}$ $\left(W^{2}=5,10\right.$ and $\left.20 \mathrm{GeV}^{2}\right)$ with the cut at $\Delta_{T \min }^{2}=-0.2 \mathrm{GeV}^{2}$. As phenomenological input, our model for $\pi N$ TDAs requires the nucleon DAs $V^{p}, A^{p}, T^{p}$ at the normalization scale $\mu^{2} \sim Q^{2}$. A vast literature exists on the phenomenological solutions for nucleon DAs (see e.g. [24, 25] for the discussion). In order to quantify the sensitivity of our model prediction on the input nucleon DAs, we show the cross section estimates for the case of several phenomenological solutions fitting the nucleon electromagnetic form 
factor: Chernyak-Ogloblin-Zhitnitsky (COZ) [26] $]^{4}$ (dashed line with long dashes), King and Sachrajda (KS) [27] $]^{5}$ (solid line), Braun-Lenz-Wittmann (BLW NLO) model of [25] ${ }^{6}$ (dashed line with medium dashes) and NNLO modification of BLW model suggested in Ref. [28] $]^{7}$ (dashed line with short dashes). The phenomenological solutions for the nucleon DA presented in the literature are usually given at a low normalization point $\mu^{2}=1 \mathrm{GeV}^{2}$, while the adequate choice of the normalization scale within our factorized approach is $\mu^{2}=Q^{2}$. In our cross section estimates presented on Figs. 4, 5 we do not take systematically into account the evolution effects which are expected to be not very significant due to the small lever arm in $Q^{2}$.

To get an estimate of these effects for the kinematics in question we show on Fig. 6 the ratio of the integrated cross sections

$$
\frac{d \bar{\sigma} /\left.d Q^{2}\right|_{\mu^{2}=Q^{2}}}{d \bar{\sigma} /\left.d Q^{2}\right|_{\mu^{2}=1 \mathrm{GeV}^{2}}}
$$

for $\bar{p} p \rightarrow \pi^{0} \ell^{+} \ell^{-}$with and without account of the evolution effects for input nucleon DA as the function of $Q^{2}$ (with $\alpha_{s}$ kept fixed). The result is presented for the BLW NNLO input nucleon DA. For $Q^{2}=2 \div 4 \mathrm{GeV}^{2}$ the effect of the evolution results in a mere $40 \%$ decrease of the cross section. This effect is much below the model dependence due to different phenomenological input. For other input phenomenological DAs we expect the effect of the evolution to be of a similar order of magnitude.

Finally we conclude that within the considered parameter range, the cross sections of $\bar{p} p \rightarrow \pi^{0} \ell^{+} \ell^{-}$and $\bar{p} n \rightarrow \pi^{-} \ell^{+} \ell^{-}$presented on Figs. 4, 5 seem to be large enough for a detailed investigation to be carried at PANDA@GSI-FAIR.

\footnotetext{
${ }^{4}$ Eq. (13) of Ref. [26].

${ }^{5}$ Eq. (4.6) of Ref. [27]

${ }^{6}$ See Appendix B of Ref. [25].

${ }^{7}$ See Table I of Ref. [28].
} 

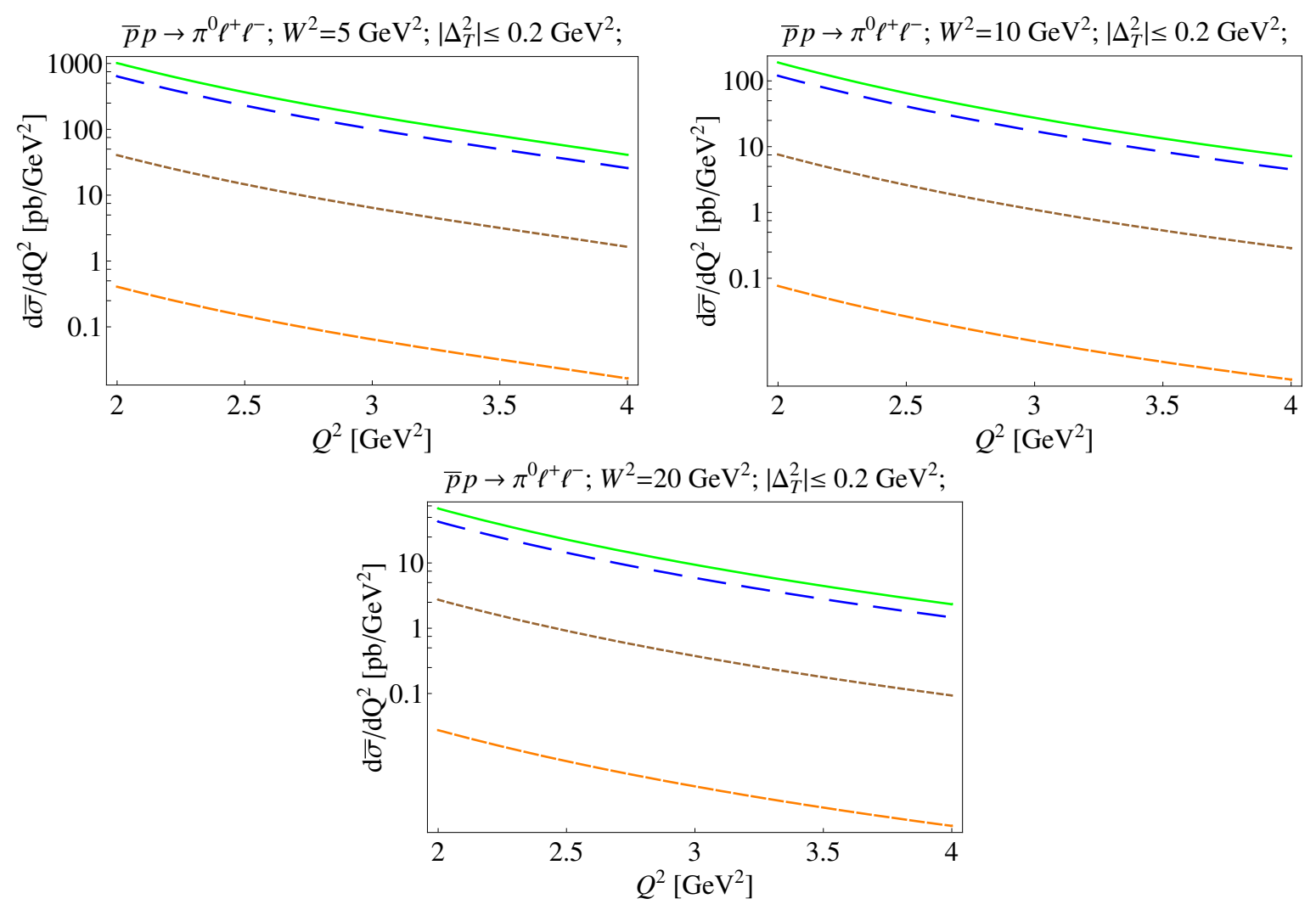

FIG. 4: Integrated cross section $d \bar{\sigma} / d Q^{2}$ for $\bar{p} p \rightarrow \ell^{+} \ell^{-} \pi^{0}$ as a function of $Q^{2}$ for different values of $W^{2}=5,10$ and $20 \mathrm{GeV}^{2}$ for various phenomenological nucleon DA solutions: COZ (long dashes); KS (solid line); BLW NLO (medium dashes) and NNLO modification [28] of BLW (short dashes).

It is worth mentioning that the cross section that we obtained with the BLW NLO model input is much smaller than that in the case of models including next-to-next-toleading conformal spin contribution (such as COZ, KS and NNLO modification of BLW). In fact, the $p \bar{p} \rightarrow \pi^{0} \gamma^{*}$ and $n \bar{p} \rightarrow \pi^{-} \gamma^{*}$ amplitudes turn to be zero at the leading twist accuracy once we employ the asymptotic form of the nucleon DA as input. This zero is reminiscent of the zero for the proton electromagnetic form factor with the asymptotic DA [10]. The cross section thus turns out to be small for the BLW NLO as well as for the Bolz-Kroll (BK) [29] input nucleon DAs which are known to be rather close to the asymptotic form of the nucleon DA. 

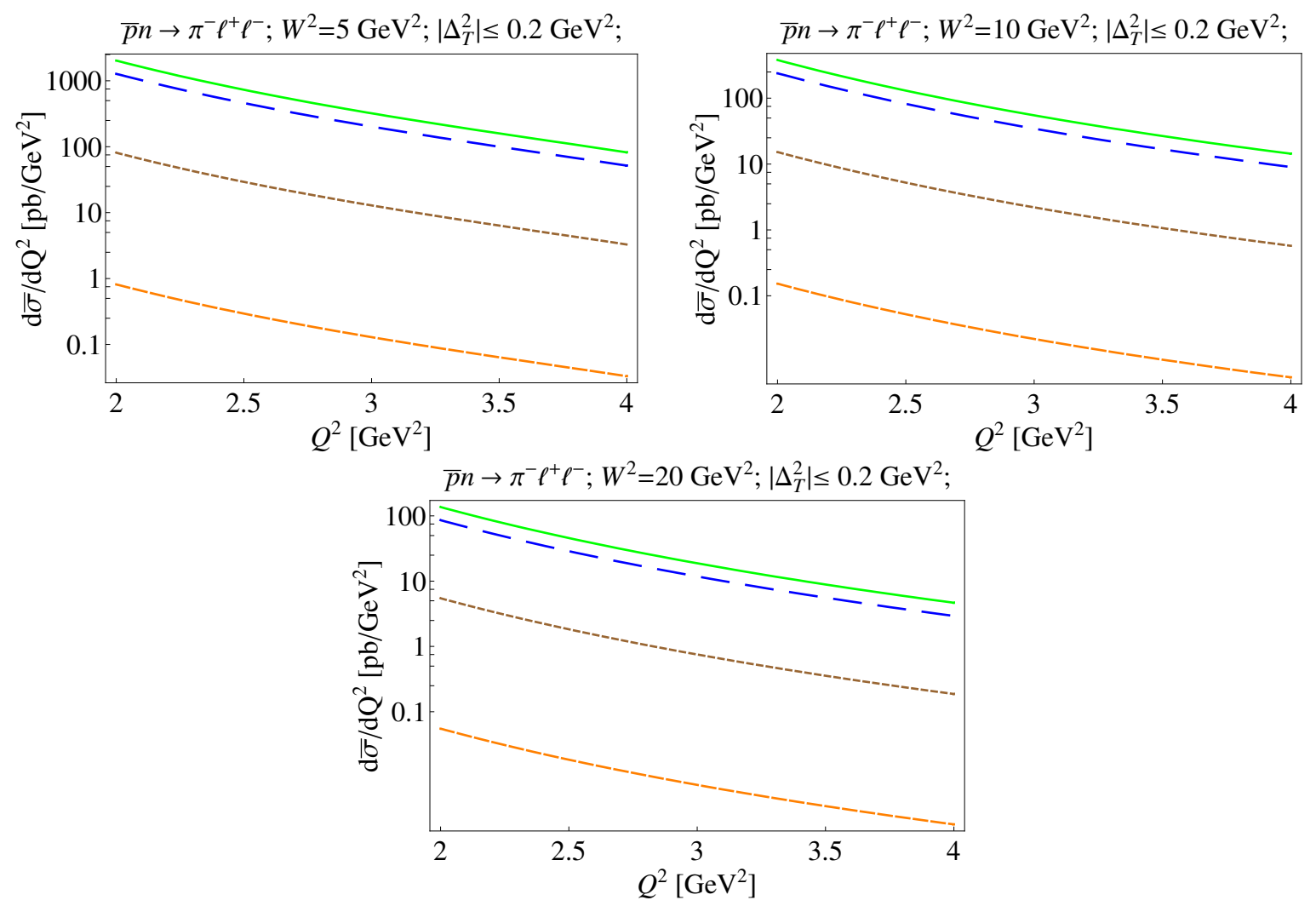

FIG. 5: Integrated cross section $d \bar{\sigma} / d Q^{2}$ for $\bar{p} n \rightarrow \ell^{+} \ell^{-} \pi^{-}$as a function of $Q^{2}$, for different values of $W^{2}=5,10$ and $20 \mathrm{GeV}^{2}$ for various phenomenological nucleon DA solutions: COZ (long dashes); KS (solid line); BLW NLO (medium dashes) and NNLO modification [28] of BLW (short dashes).

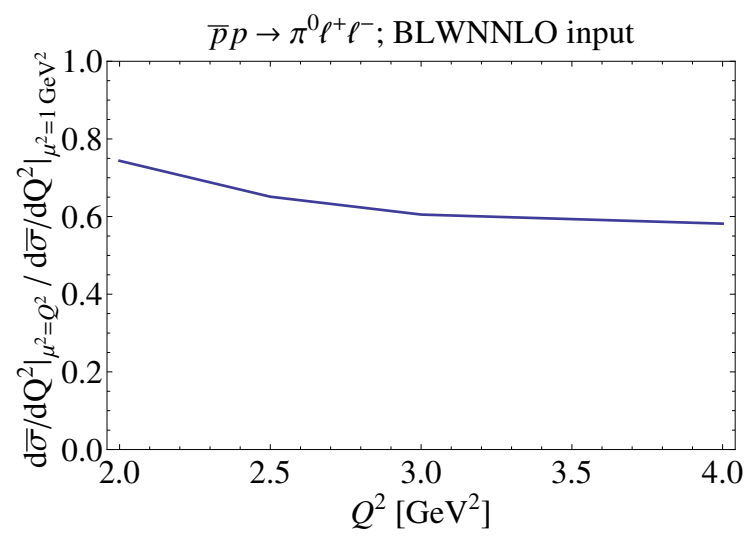

FIG. 6: The ratio of the integrated cross sections $\frac{d \bar{\sigma} /\left.d Q^{2}\right|_{\mu^{2}=Q^{2}}}{d \bar{\sigma} /\left.d Q^{2}\right|_{\mu}{ }^{2}=1 \mathrm{GeV}^{2}}$ with and without taking account of the evolution effects as a function of $Q^{2}$. 
In Fig. 77, we show the differential cross section $d \sigma / d Q^{2} d \cos \theta_{\pi}^{*}$ for $\bar{p} p \rightarrow \ell^{+} \ell^{-} \pi^{0}$ as a function of $\cos \theta_{\pi}^{*}$, for $W^{2}=5 \mathrm{GeV}^{2}$ and $Q^{2}=2.5 \mathrm{GeV}^{2}$, both for the near forward and backward factorization regimes. As a consequence of $C$-invariance, the cross section of (11) within the $t$-channel factorization mechanism can be obtained from that within the $u$-channel factorization mechanism with the change (C1) (see discussion in App. B3). The COZ solution for the nucleon DAs is used here as numerical input. The forward and backward peaks produced within the suggested factorization mechanism are clearly visible. They look perfectly symmetric in the CMS frame. However, it is worth mentioning that when boosting from the CMS to the LAB frame (which corresponds to the nucleon $N$ at rest in the $\overline{\mathrm{P}} \mathrm{ANDA}$ setup), the forward peak is narrowed and the backward peak is broadened (see Fig. 3).

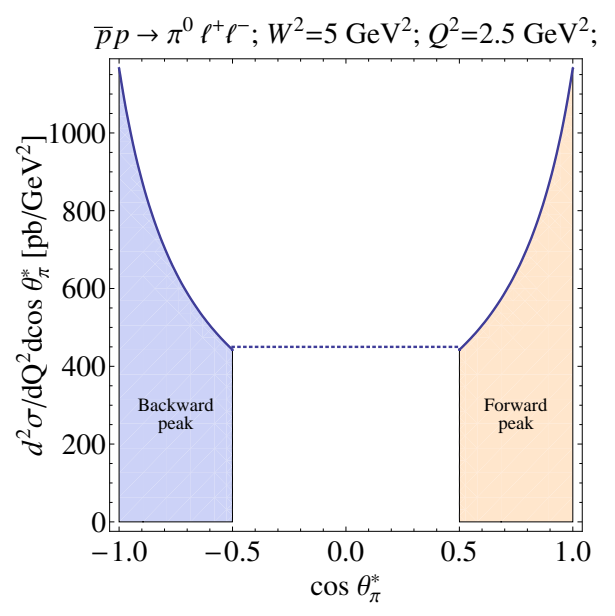

FIG. 7: Differential cross section $d \sigma / d Q^{2} d \cos \theta_{\pi}^{*}$ for $\bar{p} p \rightarrow \ell^{+} \ell^{-} \pi^{0}$ as a function of $\cos \theta_{\pi}^{*}$ for $W^{2}=5 \mathrm{GeV}^{2}$ and $Q^{2}=2.5 \mathrm{GeV}^{2}$. Forward and backward peaks are clearly visible. COZ solution for the nucleon DAs is used as the numerical input. Dotted region denotes scattering over large angles in which the present factorization description does not apply.

Obviously, the $\pi N$ TDAs in the nucleon pole model have purely ERBL support and turn into zero at the borders of the ERBL domain. In a general model in which $\pi N$ TDAs do not vanish at the borders of the ERBL domain (so-called crossover trajectories $w=-\xi$, $v= \pm \xi^{\prime}$ ), important contributions into the cross section may come from the convolutions with coefficient functions that are highly singular at the crossover trajectories.

To estimate this effect we consider the two component model for $\pi N$ TDAs [5] which 
includes the spectral part (formulated in terms of quadruple distributions) and the nucleon pole part, which is the analogue of the $D$-term familiar from the GPD case. The spectral part is nonzero in both the ERBL and the Dokshitzer-Gribov-Lipatov-Altarelli-Parisi (DGLAP)-like domains and does not vanish at the cross over trajectories. This model is an attempt to maximally take into account the general requirements (following from the underlaying fundamental field theory), such as the following:

- polynomiality property and the support property which are the consequences of the Lorentz invariance;

- permutation symmetry and the isotopic invariance;

- chiral properties manifest through the soft pion theorem.

Nevertheless the two component model of Ref. [5] is still very flexible. In particular, the factorized Ansatz for the quadruple distributions and the shape of the profile function are ad hoc assumptions. So we can rather make a model dependent estimate of the contributions of the cross over trajectories and of the DGLAP region into the cross section. As the nucleon pole contribution dies out for $\xi=1$, the spectral part contribution becomes relatively more important for higher $\xi$.

In Fig. 8 we show the effect of adding the contribution of the spectral part for the integrated cross section $d \bar{\sigma} / d Q^{2}$ of $p \bar{p} \rightarrow \pi^{0} \ell^{+} \ell^{-}$presented inn Fig. 4. Thin dashes show the pure nucleon pole part contribution; thick dashed lines show the sum (at the amplitude level) of two contributions. The COZ phenomenological solution for the nucleon DA is used as the numerical input. For different phenomenological inputs the effect of adding the spectral part is similar. 

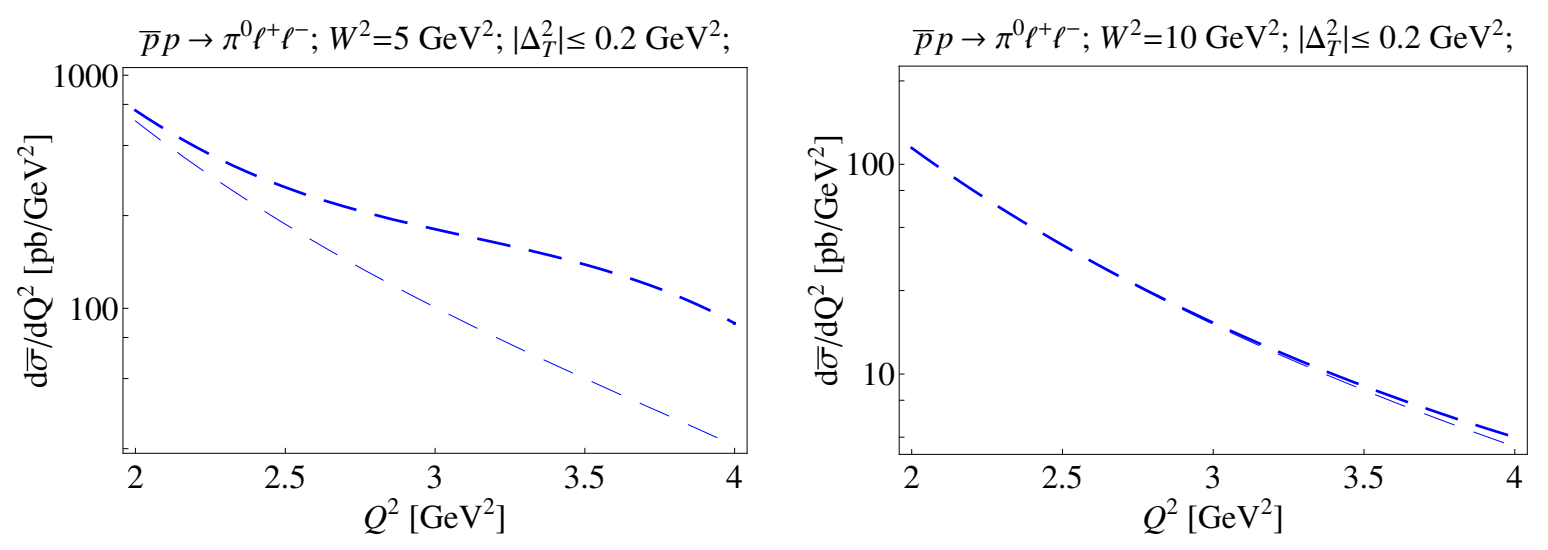

FIG. 8: The integrated cross section $d \bar{\sigma} / d Q^{2}$ of $\bar{p} p \rightarrow \ell^{+} \ell^{-} \pi^{0}$ as a function of $Q^{2}$ for $W^{2}=5 \mathrm{GeV}^{2}$ (left panel) and $W^{2}=10 \mathrm{GeV}^{2}$ (right panel) in the two component model of $\pi N$ TDAs (thick dashed line) v.s. the nucleon pole model (thin dashed line). COZ nucleon DA is used as the numerical input.

One may see that for $W^{2}=5 \mathrm{GeV}^{2}$, adding the contribution of the spectral part results, at most, in a factor of 2 increase of the cross section for $Q^{2} \sim 4 \mathrm{GeV}^{2}$ (this kinematical region corresponds to rather large values of $\xi \sim 0.6$ ). This effect is not very important in view of the large uncertainty due to different input phenomenological DAs. Therefore the account of the spectral part contribution together with the question of appropriate modelling of quadruple distributions will become a topical question after a stable signal from the backward (and forward) regimes will be available from PANDA. Moreover, for $W^{2}=10 \mathrm{GeV}^{2}$ the $Q^{2}$ interval in question corresponds to rather small values of $\xi \sim 0.1$, for which the nucleon pole part totally dominates over the spectral part within the present model.

\section{Results for $\eta$ production}

In an analogous way, we may consider near backward (and forward) $\eta$ meson production in association with a lepton pair

$$
N\left(p_{p}\right)+\bar{N}\left(p_{\bar{p}}\right) \rightarrow \gamma^{*}(q)+\eta\left(p_{\eta}\right) \rightarrow \ell^{+}\left(p_{\ell^{+}}\right)+\ell^{-}\left(p_{\ell^{-}}\right)+\eta\left(p_{\eta}\right)
$$

The nucleon pole model for $\eta N$ TDAs is similar to that for $\pi N$ TDAs (44) with the obvious change of phenomenological coupling $g_{\pi N N} \rightarrow g_{\eta N N}$. Estimates of $g_{\pi N N}$ and 
$g_{\eta N N}$ phenomenological couplings taken from Table 9.1 of Ref. [30] give $\frac{g_{\eta N N}^{2}}{g_{\pi N N}^{2}} \sim 0.3$. The formulas for the $\bar{p} p \rightarrow \gamma^{*} \eta \rightarrow \ell^{+} \ell^{-} \eta$ are obtained from the relevant formulas of Sec. 3 with the obvious change of values of the masses and the couplings. In Fig. 9, we show the results for the integrated cross section $d \sigma^{\text {int }} / d Q^{2}$ for $\bar{p} p \rightarrow \ell^{+} \ell^{-} \eta$ as a function of $Q^{2}$, for different $W^{2}=5,10$ and $20 \mathrm{GeV}^{2}$ for various phenomenological nucleon DA solutions: COZ (long dashes), KS (solid line), BLW NLO (medium dashes) and NNLO modification [28] of BLW model (short dashes).

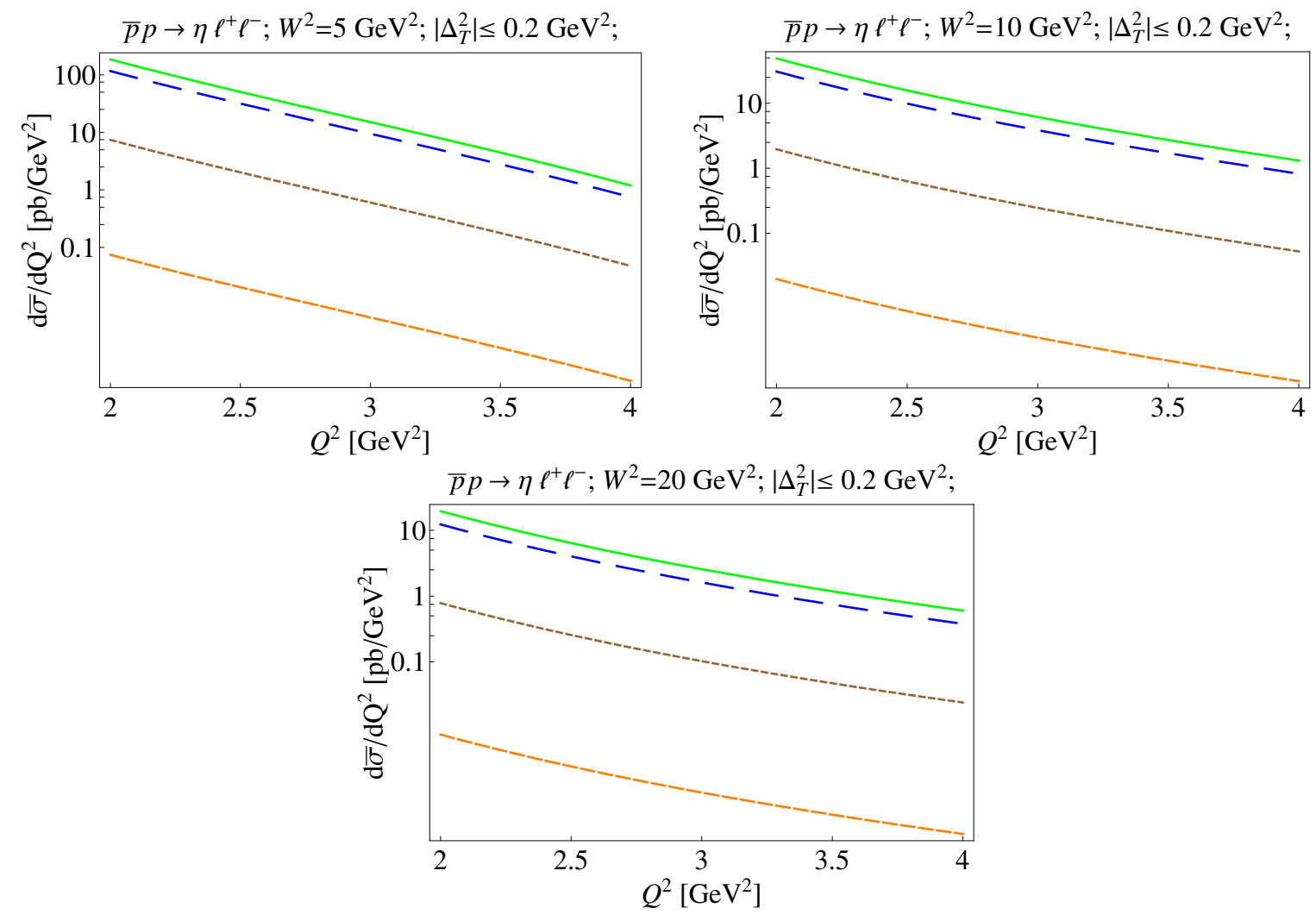

FIG. 9: Integrated cross section $d \bar{\sigma} / d Q^{2}$ for $\bar{p} p \rightarrow \ell^{+} \ell^{-} \eta$ as a function of $Q^{2}$ for different values of $W^{2}=5,10$ and $20 \mathrm{GeV}^{2}$ for various phenomenological nucleon DA solutions: COZ (long dashes); KS (solid line); BLW NLO (medium dashes) and NNLO modification [28] of BLW (short dashes). 


\section{CONCLUSIONS}

In this work, we have estimated the cross section for pseudoscalar meson production in association with a high invariant mass lepton pair in nucleon antinucleon annihilation. We have performed this analysis in the framework of the QCD collinear factorization. We used the model for the TDAs which was introduced recently in [15] and for which the major input is the parametrization of the nucleon distribution amplitudes. For most of the choices, the magnitudes of the cross sections are sufficient to be measurable with the luminosity foreseen at the PANDA experiment at GSI-FAIR. On the other hand, it turns out that the cross section depends much on the parametrization used for these DAs, which may be analyzed in a positive way as an indication that the experimental study of these processes will help to distinguish between the different models for these DAs. Note that these different models all claim to be in reasonable agreement with the nucleon form factor measurements. We are, however, still far from proposing a strategy for a model-independent extraction of the TDAs from experimental data. More observables, and in particular spin asymmetries, should be discussed. Coefficient functions should also be calculated at next-to-leading order and the effect of the $Q^{2}$ evolution of the TDAs should also be studied in details. Note that these NLO order effects are known to be different in timelike and spacelike processes [31]. Meanwhile, the preparation for the experimental analysis requires detailed background studies (such as that of the final states where a $\pi^{+} \pi^{-}$meson pair mimics the lepton pair) and a careful estimation of detector efficiency. Although we concentrated here on pseudoscalar ( $\pi$ and $\eta$ ) meson production, this framework can be extended to the production of vector mesons $(\rho, \omega)$ which will uncover complementary aspects of hadronic structure both experimentally and theoreti-

cally. Also a purely hadronic description of $\bar{p} p \rightarrow \pi \ell^{+} \ell^{-}$is proposed in Refs. [32] and [33], respectively, in a Born model and a Regge model description of baryonic exchanges.

\section{Acknowledgements}

We are thankful to Thierry Hennino, Frank Maas and Manuel Zambrana for constructive and motivating discussions. This work is partly supported by the Polish Grant NCN No. DEC-2011/01/B/ST2/03915, the French-Polish collaboration agreement Polonium, 
the P2IO consortium and the Joint Research Activity Study of Strongly Interacting Matter (acronym HadronPhysics3, Grant Agreement n.283286) under the Seventh Framework Programme of the European Community and by the COPIN-IN2P3 Agreement.

\section{A. KINEMATICS}

Below we review the relevant kinematical quantities discussed in [4] for the two possible factorization regimes for the reaction (11) involving nucleon to meson and antinucleon to meson TDAs.

\section{$u$-channel factorization regime}

Within the conventions natural for the PANDA setup, the $u$-channel factorization regime (see the left panel of Fig. 1) in which $|u|$ is small corresponds to the meson moving in the direction of the initial proton (backward direction).

The $z$-axis is chosen along the colliding nucleon-antinucleon. We define the light-cone vectors $p^{u}$ and $n^{u}$ such that $2 p^{u} \cdot n^{u}=1$. We introduce the average momentum and $u$-channel momentum transfer

$$
P^{u}=\frac{1}{2}\left(p_{\mathcal{M}}+p_{N}\right) ; \quad \Delta^{u}=p_{\mathcal{M}}-p_{N} ; \quad \Delta^{u 2} \equiv u .
$$

$\Delta^{u}{ }_{T}$ is the transverse component of $\Delta^{u}\left(\Delta_{T}^{u} \leq 0\right)$. We define the skewness variable

$$
\xi^{u}=-\frac{\Delta^{u} \cdot n^{u}}{2 P^{u} \cdot n^{u}}
$$

The following Sudakov decomposition is valid for the momenta of the reaction (7):

$$
\begin{aligned}
& p_{N}=\left(1+\xi^{u}\right) p^{u}+\frac{M^{2}}{1+\xi^{u}} n^{u} ; \\
& p_{\bar{N}}=\frac{2 M^{2}\left(1+\xi^{u}\right)}{W^{2}-2 M^{2}+W \sqrt{W^{2}-4 M^{2}}} p^{u}+\frac{W^{2}-2 M^{2}+W \sqrt{W^{2}-4 M^{2}}}{2\left(1+\xi^{u}\right)} n^{u} ; \\
& p_{\mathcal{M}}=\left(1-\xi^{u}\right) p^{u}+\frac{m^{2}-\Delta_{T}^{u}}{1-\xi^{u}} n^{u}+\Delta_{T}^{u} ; \\
& \Delta^{u}=-2 \xi^{u} p^{u}+\left[\frac{m^{2}-\Delta_{T}^{u} 2}{1-\xi^{u}}-\frac{M^{2}}{1+\xi^{u}}\right] n^{u}+\Delta_{T}^{u} ; \\
& q=\left[2 \xi^{u}+\frac{M^{2}}{W^{2}}\left(1+\xi^{u}\right)+O\left(1 / W^{4}\right)\right] p^{u}+\left[\frac{W^{2}-M^{2}}{1+\xi^{u}}-\frac{m^{2}-\Delta_{T}^{u}}{1-\xi^{u}}+O\left(1 / W^{2}\right)\right] n^{u}-\Delta_{T}^{u},
\end{aligned}
$$


where $M(m)$ denotes nucleon (meson) mass.

In the nucleon rest frame (which corresponds to the PANDA laboratory frame) the light-cone vectors $p^{u}$ and $n^{u}$ read

$$
\left.p^{u}\right|_{N \text { rest }}=\frac{M}{2\left(1+\xi^{u}\right)}\{1,0,0,-1\} ;\left.\quad n^{u}\right|_{N \text { rest }}=\frac{1+\xi^{u}}{2 M}\{1,0,0,1\} .
$$

With the help of the appropriate boost we establish the expressions for the light-cone vectors in the $N \bar{N}$ CMS

$$
\left.p^{u}\right|_{\bar{N} N \mathrm{CMS}}=\left\{\alpha^{u}, 0,0,-\alpha^{u}\right\} ;\left.\quad n^{u}\right|_{\bar{N} N \mathrm{CMS}}=\left\{\beta^{u}, 0,0, \beta^{u}\right\},
$$

where

$$
\alpha^{u}=\frac{W+\sqrt{W^{2}-4 M^{2}}}{4\left(1+\xi^{u}\right)} ; \quad \beta^{u}=\frac{\left(W-\sqrt{W^{2}-4 M^{2}}\right)\left(1+\xi^{u}\right)}{4 M^{2}} .
$$

The meson $\mathcal{M}$ scattering angle in the $N \bar{N}$ CMS for the $u$-channel factorization regime then can be expressed as:

$$
\cos \theta_{\mathcal{M}}^{*}=\frac{-\left(1-\xi^{u}\right) \alpha^{u}+\frac{m^{2}-\Delta_{T}^{u} 2}{1-\xi^{u}} \beta^{u}}{\sqrt{\left(-\left(1-\xi^{u}\right) \alpha^{u}+\frac{m^{2}-\Delta_{T}^{u 2}}{1-\xi^{u}} \beta^{u}\right)^{2}-\Delta_{T}^{u 2}}} .
$$

One may check that for $\Delta_{T}^{u 2}=0$ indeed $\cos \theta_{\mathcal{M}}^{*}=-1$, which means backward scattering.

We also quote some useful relations for the kinematical quantities:

$$
\begin{aligned}
& \Delta_{T}^{u 2}=\frac{1-\xi^{u}}{1+\xi^{u}}\left(u-2 \xi^{u}\left[\frac{M^{2}}{1+\xi^{u}}-\frac{m^{2}}{1-\xi^{u}}\right]\right) ; \\
& Q^{2} \equiv q^{2}=\frac{2 \xi^{u}}{1+\xi^{u}} W^{2}+u-3 M^{2}+\frac{4 M^{2}}{1+\xi^{u}}+O\left(1 / W^{2}\right) ; \\
& t=\left(p_{\mathcal{M}}-p_{\bar{N}}\right)^{2}=-\frac{1-\xi^{u}}{1+\xi^{u}} W^{2}+m^{2}-M^{2}+\frac{4 M^{2}}{1+\xi^{u}}+O\left(1 / W^{2}\right) .
\end{aligned}
$$

This allows to express $\xi^{u}$ as

$$
\xi^{u} \simeq \frac{Q^{2}-u-M^{2}}{2 W^{2}-Q^{2}+u-3 M^{2}}
$$

\section{$t$-channel factorization regime}

The $t$-channel factorization regime (see the right panel of Fig. 10 in which $|t|$ is small corresponds to the meson moving in the direction of the initial antiproton (forward direction). 
We define the light-cone vectors $p^{t}$ and $n^{t}$ such that $2 p^{t} \cdot n^{t}=1$.

$$
P^{t}=\frac{1}{2}\left(p_{\mathcal{M}}+p_{\bar{N}}\right) ; \quad \Delta^{t}=p_{\mathcal{M}}-p_{\bar{N}} ; \quad \Delta^{t^{2}} \equiv t .
$$

$\Delta^{t}{ }_{T}$ is the transverse component of $\Delta^{t}\left(\Delta_{T}^{t} \cdot \Delta_{T}^{t}=\Delta_{T}^{t}{ }^{2} \leq 0\right)$. We define the skewness variable

$$
\xi^{t}=-\frac{\Delta^{t} \cdot n^{t}}{2 P^{t} \cdot n^{t}}
$$

The following Sudakov decomposition is valid for the momenta of particles:

$$
\begin{aligned}
& p_{\bar{N}}=\left(1+\xi^{t}\right) p^{t}+\frac{M^{2}}{1+\xi^{t}} n^{t} ; \\
& p_{N}=\frac{2 M^{2}\left(1+\xi^{t}\right)}{W^{2}-2 M^{2}+W \sqrt{W^{2}-4 M^{2}}} p^{t}+\frac{W^{2}-2 M^{2}+W \sqrt{W^{2}-4 M^{2}}}{2\left(1+\xi^{t}\right)} n^{t} ; \\
& p_{\mathcal{M}}=\left(1-\xi^{t}\right) p^{t}+\frac{m^{2}-\Delta_{T}^{t}}{1-\xi^{t}} n^{t}+\Delta_{T}^{t} ; \\
& \Delta^{t}=-2 \xi^{t} p^{t}+\left[\frac{m^{2}-\Delta_{T}^{t}}{1-\xi^{t}}-\frac{M^{2}}{1+\xi^{t}}\right] n^{t}+\Delta_{T}^{t} ; \\
& q=\left[2 \xi^{t}+\frac{M^{2}}{W^{2}}\left(1+\xi^{t}\right)+O\left(1 / W^{4}\right)\right] p^{t}+\left[\frac{W^{2}-M^{2}}{1+\xi^{t}}-\frac{m^{2}-\Delta_{T}^{t}}{1-\xi^{t}}+O\left(1 / W^{2}\right)\right] n^{t}-\Delta_{T}^{t} .
\end{aligned}
$$

In the antinucleon rest frame the light-cone vectors $p^{t}$ and $n^{t}$ are

$$
\left.p^{t}\right|_{\bar{N} \text { rest }}=\frac{M}{2\left(1+\xi^{t}\right)}\{1,0,0,1\} ;\left.\quad n^{t}\right|_{\bar{N} \text { rest }}=\frac{1+\xi^{t}}{2 M}\{1,0,0,-1\} .
$$

The explicit expressions for the light-cone vectors $p^{t}$ and $n^{t}$ in the $\bar{N} N$ CMS read:

$$
\left.p^{t}\right|_{\bar{N} N \text { CMS }}=\left\{\alpha^{t}, 0,0, \alpha^{t}\right\} ;\left.\quad n^{t}\right|_{\bar{N} N \text { CMS }}=\left\{\beta^{t}, 0,0,-\beta^{t}\right\},
$$

where $\alpha^{t}$ and $\beta^{t}$ are defined as

$$
\alpha^{t}=\frac{W+\sqrt{W^{2}-4 M^{2}}}{4\left(1+\xi^{t}\right)} ; \quad \beta^{t}=\frac{\left(W-\sqrt{W^{2}-4 M^{2}}\right)\left(1+\xi^{t}\right)}{4 M^{2}} .
$$

The meson $\mathcal{M}$ scattering angle in the $N \bar{N}$ CMS for the $t$-channel factorization regime then expresses as:

$$
\cos \theta_{\mathcal{M}}^{*}=\frac{\left(1-\xi^{t}\right) \alpha^{t}-\frac{m^{2}-\Delta_{T}^{t}}{1-\xi^{t}} \beta^{t}}{\sqrt{\left(\left(1-\xi^{t}\right) \alpha^{t}-\frac{m^{2}-\Delta_{T}^{t}}{1-\xi^{t}} \beta^{t}\right)^{2}-\Delta_{T}^{t 2}}} .
$$


Again one may check that for $\Delta_{T}^{t^{2}}=0$, indeed, $\cos \theta_{\mathcal{M}}^{*}=1$, which means forward scattering.

We also work out the relations:

$$
\begin{aligned}
& \Delta_{T}^{t^{2}}=\frac{1-\xi^{t}}{1+\xi^{t}}\left(t-2 \xi^{t}\left[\frac{M^{2}}{1+\xi^{t}}-\frac{m^{2}}{1-\xi^{t}}\right]\right) ; \\
& Q^{2} \equiv q^{2}=\frac{2 \xi^{t}}{1+\xi^{t}} W^{2}+t-3 M^{2}+\frac{4 M^{2}}{1+\xi^{t}}+O\left(1 / W^{2}\right) ; \\
& u=\left(p_{\mathcal{M}}-p_{N}\right)^{2}=-\frac{1-\xi^{t}}{1+\xi^{t}} W^{2}+m^{2}-M^{2}+\frac{4 M^{2}}{1+\xi^{t}}+O\left(1 / W^{2}\right) .
\end{aligned}
$$

and express $\xi^{t}$ :

$$
\xi^{t} \simeq \frac{Q^{2}-t-M^{2}}{2 W^{2}-Q^{2}+t-3 M^{2}} .
$$

\section{B. CHARGE CONJUGATION AND G-PARITY ISSUES}

\section{Notations and conventions}

Below we employ our usual system notations of Ref. [15].

- Letters from the beginning of the Greek alphabet are reserved for the $\mathrm{SU}(2)$ isospin indices $\alpha, \beta, \gamma, \iota, \kappa=1,2$.

- We have to distinguish between upper (contravariant) and lower (covariant) SU(2) isospin indices. We introduce the totally antisymmetric tensor $\varepsilon_{\alpha \beta}$ for lowering indices and $\varepsilon^{\alpha \beta}$ for rising indices $\left(\varepsilon_{12}=\varepsilon^{12}=1\right)$.

- Letters from the middle of the Greek alphabet $\lambda, \mu, \nu$ denote the Lorentz indices.

- Letters from the second half of the Greek alphabet $\rho, \tau, \chi$ are reserved for the Dirac indices.

- Letters from the beginning of the Latin alphabet $a, b, c \ldots$ are reserved for indices of the adjoint representation of the $\mathrm{SU}(2)$ isospin group.

- Letters $c_{1}, c_{2}, c_{3}$ stand for $\mathrm{SU}(3)$ color indices. 
The nucleon field $\bar{N}_{\alpha}\left(N^{\alpha}\right)$ transforms according to the covariant (contravariant) representation of the isospin $\mathrm{SU}(2)$. We adopt the following standard convention for the nucleon field [34]:

$$
\begin{aligned}
& N^{\alpha}(x)=\int \frac{d^{3} k}{(2 \pi)^{3}} \frac{M}{k_{0}} \sum_{s=1,2}\left\{e^{i k x} d^{\dagger \alpha}(k, s) V(k, s)+e^{-i k x} b^{\alpha}(k, s) U(k, s)\right\} ; \\
& \bar{N}_{\alpha}(x)=\int \frac{d^{3} k}{(2 \pi)^{3}} \frac{M}{k_{0}} \sum_{s=1,2}\left\{e^{i k x} b_{\alpha}^{\dagger}(k, s) \bar{U}(k, s)+e^{-i k x} d_{\alpha}(k, s) \bar{V}(k, s)\right\} .
\end{aligned}
$$

Here spinors $U(k, s)$ and $\bar{U}(k, s) \equiv U^{\dagger}(k, s) \gamma_{0}$ describe a nucleon, respectively, in the initial and final states, while spinors $\bar{V}(k, s) \equiv V^{\dagger}(k, s) \gamma_{0}$ and $V(k, s)$ describe an antinucleon in the initial and final states.

The creation and annihilation operators in (B1) satisfy the usual anticommutation relations for fermions

$$
\begin{aligned}
& \left\{b^{\alpha}(p, s), b^{\dagger}{ }_{\beta}\left(p^{\prime}, s^{\prime}\right)\right\}=(2 \pi)^{3} \frac{p_{0}}{M} \delta^{3}\left(p-p^{\prime}\right) \delta_{s s^{\prime}} \delta_{\beta}^{\alpha} ; \\
& \left\{d_{\alpha}(p, s), d^{\dagger \beta}\left(p^{\prime}, s^{\prime}\right)\right\}=(2 \pi)^{3} \frac{p_{0}}{M} \delta^{3}\left(p-p^{\prime}\right) \delta_{s s^{\prime}} \delta_{\alpha}^{\beta} .
\end{aligned}
$$

The "in" nucleon state $\left|N_{\alpha}\right\rangle$ is defined according to:

$$
\left|N_{1}\right\rangle \equiv\left|N_{p}(p, s)\right\rangle=b_{1}^{\dagger}(p, s)|0\rangle ; \quad\left|N_{2}\right\rangle \equiv\left|N_{n}(p, s)\right\rangle=b_{2}^{\dagger}(p, s)|0\rangle
$$

Analogously, the "in" antiparticle state $\left|\bar{N}^{\alpha}\right\rangle$ is defined as:

$$
\left|\bar{N}^{1}\right\rangle \equiv\left|N^{\bar{p}}(p, s)\right\rangle=d^{\dagger 1}(p, s)|0\rangle ; \quad\left|\bar{N}^{2}\right\rangle \equiv\left|N^{\bar{n}}(p, s)\right\rangle=d^{\dagger 2}(p, s)|0\rangle .
$$

The charge conjugation operator $\mathcal{C}$ has the following effect on the nucleon creation and antinucleon annihilation operators [34]

$$
\begin{aligned}
& \mathcal{C} b^{\iota}(p, \lambda) \mathcal{C}^{\dagger}=\eta_{N} d_{\iota}(p, \lambda) ; \\
& \mathcal{C} d^{\dagger \iota}(p, \lambda) \mathcal{C}^{\dagger}=\eta_{N} b_{\iota}^{\dagger}(p, \lambda),
\end{aligned}
$$

where $\eta_{N}$ denotes the nucleon field charge parity. These equalities are to be understood not as equalities of $\mathrm{SU}(2)$ tensors but rather as equalities for their components. From $(\overline{\mathrm{B} 6})$ and the hermitian conjugation of $(\overline{\mathrm{B} 5})$, we get, respectively:

$$
\begin{aligned}
& \mathcal{C}\left|\bar{N}^{\iota}\right\rangle=\eta_{N}\left|N_{\iota}\right\rangle ; \\
& \mathcal{C}\left|N_{\iota}\right\rangle=\eta_{N}^{*}\left|\bar{N}^{\iota}\right\rangle .
\end{aligned}
$$




\section{Antinucleon DA}

The isotopic parametrization for the nucleon DA reads [15]

$$
\begin{aligned}
& 4\left\langle 0\left|\varepsilon_{c_{1} c_{2} c_{3}} \Psi_{\rho}^{c_{1} \alpha}(1) \Psi_{\tau}^{c_{2} \beta}(2) \Psi_{\chi}^{c_{3} \gamma}(3)\right| N_{\iota}(p)\right\rangle \\
& =\varepsilon^{\alpha \beta} \delta_{\iota}^{\gamma} M_{\rho \tau \chi}^{N\{13\}}(1,2,3)+\varepsilon^{\alpha \gamma} \delta_{\iota}^{\beta} M_{\rho \tau \chi}^{N\{12\}}(1,2,3),
\end{aligned}
$$

where the invariant isospin amplitudes read

$$
\begin{aligned}
& M_{\rho \tau \chi}^{N\{12\}}(1,2,3)=f_{N}\left(v_{\rho \tau, \chi}^{N} V^{p}(1,2,3)+a_{\rho \tau, \chi}^{N} A^{p}(1,2,3)+t_{\rho \tau, \chi}^{N} T^{p}(1,2,3)\right) \\
& M_{\rho \tau \chi}^{N\{13\}}(1,2,3)=M_{\rho \chi \tau}^{N\{12\}}(1,3,2) .
\end{aligned}
$$

Here $\left\{v^{N}, a^{N}, t^{N}\right\}_{\rho \tau, \chi}$ are the conventional Dirac structures,

$$
v_{\rho \tau, \chi}^{N}=(\hat{p} C)_{\rho \tau}\left(\gamma^{5} U(p)\right)_{\chi} ; \quad a_{\rho \tau, \chi}^{N}=\left(\hat{p} \gamma^{5} C\right)_{\rho \tau}(U(p))_{\chi} ; \quad t_{\rho \tau, \chi}^{N}=\left(\sigma_{p \mu} C\right)_{\rho \tau}\left(\gamma^{\mu} \gamma^{5} U(p)\right)_{\chi}
$$

where $C$ is the charge conjugation matrix; $\sigma^{\mu \nu}=\frac{1}{2}\left[\gamma^{\mu}, \gamma^{\nu}\right]$ and $\sigma^{p \nu} \equiv p_{\mu} \sigma^{\mu \nu}$.

The effect of the charge conjugation operator on the quark field $\Psi^{\alpha}$ is [34]:

$$
\mathcal{C} \Psi^{\alpha} \mathcal{C}^{\dagger}=\eta_{q} C \bar{\Psi}_{\alpha}^{T}
$$

where transposition refers to the Dirac index. $C$ stands for the charge conjugation matrix and $\eta_{q}$ is the corresponding charge parity.

Now using (B11) together with (B7), we establish the link between nucleon and antinucleon DAs,

$$
\begin{aligned}
& 4\left\langle 0\left|\varepsilon_{c_{1} c_{2} c_{3}} \Psi_{\rho}^{c_{1} \alpha}(1) \Psi_{\tau}^{c_{2} \beta}(2) \Psi_{\chi}^{c_{3} \gamma}(3)\right| N_{\iota}(p)\right\rangle \\
& =4 \eta_{N}^{*}\left\langle 0\left|\varepsilon_{c_{1} c_{2} c_{3}} \mathcal{C}^{\dagger} \mathcal{C} \Psi_{\rho}^{c_{1} \alpha}(1) \mathcal{C}^{\dagger} \mathcal{C} \Psi_{\tau}^{c_{2} \beta}(2) \mathcal{C}^{\dagger} \mathcal{C} \Psi_{\chi}^{c_{3} \gamma}(3) \mathcal{C}^{\dagger}\right| \bar{N}^{\iota}(p)\right\rangle \\
& =4 \eta_{N}^{*} \eta_{q}^{3}\left\langle 0\left|\varepsilon_{c_{1} c_{2} c_{3}}\left(C \bar{\Psi}_{\alpha}^{T c_{1}}\right)_{\rho}\left(C \bar{\Psi}_{\beta}^{T c_{2}}\right)_{\tau}\left(C \bar{\Psi}_{\gamma}^{T c_{3}}\right)_{\chi}\right| \bar{N}^{\iota}(p)\right\rangle .
\end{aligned}
$$

Now we can define the antinucleon DA as the $\mathrm{SU}(2)$ tensor:

$$
4\left\langle 0\left|\varepsilon_{c_{1} c_{2} c_{3}}\left(\bar{\Psi}_{\alpha}^{c_{1}}\right)_{\rho}\left(\bar{\Psi}_{\beta}^{c_{2}}\right)_{\tau}\left(\bar{\Psi}_{\gamma}^{c_{3}}\right)_{\chi}\right| \bar{N}^{\iota}(p)\right\rangle=\varepsilon_{\alpha \beta} \delta_{\gamma}^{\iota} M_{\rho \tau \chi}^{\bar{N}\{13\}}(1,2,3)+\varepsilon_{\alpha \gamma} \delta_{\beta}^{\iota} M_{\rho \tau \chi}^{\bar{N}\{12\}}(1,2,3),
$$


where

$$
\begin{aligned}
& M_{\rho \tau \chi}^{\bar{N}\{12\}}(1,2,3)=f_{N}\left(v_{\rho \tau, \chi}^{\bar{N}} V^{\bar{p}}(1,2,3)+a_{\rho \tau, \chi}^{\bar{N}} A^{\bar{p}}(1,2,3)+t_{\rho \tau, \chi}^{\bar{N}} T^{\bar{p}}(1,2,3)\right) ; \\
& M_{\rho \tau \chi}^{\bar{N}\{13\}}(1,2,3)=M_{\rho \chi \tau}^{\bar{N}\{12\}}(1,3,2),
\end{aligned}
$$

where the relevant Dirac structures are

$$
\begin{aligned}
& \left(v_{\rho \tau, \chi}^{\bar{N}}\right)^{T} \equiv\left(C^{\dagger}\right)_{\rho \rho^{\prime}}\left(C^{\dagger}\right)_{\tau \tau^{\prime}}\left(C^{\dagger}\right)_{\chi \chi^{\prime}} v_{\rho^{\prime} \tau^{\prime}, \chi^{\prime}}^{N}=(C \hat{p})_{\rho \tau}^{T}\left(\bar{V} \gamma^{5}\right)_{\chi}^{T} \\
& \left(a_{\rho \tau, \chi}^{\bar{N}}\right)^{T} \equiv\left(C^{\dagger}\right)_{\rho \rho^{\prime}}\left(C^{\dagger}\right)_{\tau \tau^{\prime}}\left(C^{\dagger}\right)_{\chi \chi^{\prime}} a_{\rho^{\prime} \tau^{\prime}, \chi^{\prime}}^{N}=\left(C \gamma_{5} \hat{p}\right)_{\rho \tau}^{T}(\bar{V})_{\chi}^{T} \\
& \left(t_{\rho \tau, \chi}^{\bar{N}}\right)^{T} \equiv\left(C^{\dagger}\right)_{\rho \rho^{\prime}}\left(C^{\dagger}\right)_{\tau \tau^{\prime}}\left(C^{\dagger}\right)_{\chi \chi^{\prime}} t_{\rho^{\prime} \tau^{\prime}, \chi^{\prime}}^{N}=\left(C \sigma_{p \mu}\right)_{\rho \tau}^{T}\left(\bar{V} \gamma^{\mu} \gamma_{5}\right)_{\chi}^{T}
\end{aligned}
$$

Lifting the transposition with respect to the Dirac indices in (B15) one gets

$$
\begin{aligned}
& v_{\rho \tau, \chi}^{\bar{N}}=(C \hat{p})_{\rho \tau}\left(\bar{V} \gamma^{5}\right)_{\chi} ; \\
& a_{\rho \tau, \chi}^{\bar{N}}=\left(C \hat{p} \gamma_{5}\right)_{\rho \tau}(\bar{V})_{\chi} ; \\
& t_{\rho \tau, \chi}^{\bar{N}}=\left(C \sigma_{p \mu}\right)_{\rho \tau}\left(\bar{V} \gamma^{\mu} \gamma_{5}\right)_{\chi} .
\end{aligned}
$$

Therefore, we conclude that as the consequence of charge conjugation invariance antiproton DAs occurring in (B14) are expressed as follows through the usual proton DAs of (B9):

$$
\{V, A, T\}^{\bar{p}}\left(y_{1}, y_{2}, y_{3}\right)=\frac{1}{\eta_{N}^{*} \eta_{q}^{3}}\{V, A, T\}^{p}\left(y_{1}, y_{2}, y_{3}\right)
$$

\section{Antinucleon to pion TDAs}

Below we establish the relations between the antinucleon to pion and nucleon to pion TDAs. Our main line of reasoning is analogous to that in the case of the antinucleon DAs. However, charged pions are not eigenstates of the charge conjugation operator $\mathcal{C}$. To work out the isotopic formalism for the antinucleon to pion TDAs it is convenient to employ the concept of $G$-parity (see e.g. [35]), which is the combination of the charge conjugation and the rotation around the second axis of the isospin space,

$$
\mathcal{G}=\mathcal{C} e^{i \pi I_{2}}
$$

First, let us work out the effect of $\mathcal{G}$ on the quark and antiquark fields $\Psi^{\alpha}$,

$$
\mathcal{G} \Psi^{\alpha} \mathcal{G}^{\dagger}=\mathcal{C} e^{i \pi I_{2}} \Psi^{\alpha} e^{-i \pi I_{2}} \mathcal{C}^{\dagger}
$$


According to the conventions of [15], we choose to transform $\bar{\Psi}_{\alpha}$ field according to the covariant representation and to transform $\Psi^{\alpha}$ field according to the contravariant representation of the isospin $\mathrm{SU}(2)$ :

$$
\left[I_{a}, \bar{\Psi}_{\alpha}\right]=\frac{1}{2}\left(\sigma_{a}\right)_{\alpha}^{\beta} \bar{\Psi}_{\beta} ; \quad\left[I_{a}, \Psi^{\alpha}\right]=-\frac{1}{2}\left(\sigma_{a}\right)_{\alpha}^{\beta} \Psi^{\alpha}
$$

where $I_{a}$ stand for the group generators and $\sigma_{a}$ are the Pauli matrices.

The effect of the isospin rotation around the second axis then reads:

$$
e^{i \pi I_{2}}\left(\Psi^{\alpha}\right)_{\rho} e^{-i \pi I_{2}}=\left(\cos \frac{\pi}{2} \delta_{\beta}^{\alpha}+\sin \frac{\pi}{2}\left(-i \sigma_{2}\right)_{\beta}^{\alpha}\right)\left(\Psi^{\beta}\right)_{\rho} .
$$

Thus, we conclude that

$$
\mathcal{G} u_{\rho} \mathcal{G}^{\dagger}=\eta_{q}(-1)(C \bar{d})_{\rho}^{T} ; \quad \mathcal{G} d_{\rho} \mathcal{G}^{\dagger}=\eta_{q}(C \bar{u})_{\rho}^{T}
$$

We also need to specify the effect of $\mathcal{G}$ on pion "out" states and nucleon "in" states. The triplet of pions has negative $G$ parity,

$$
\langle\pi| \mathcal{G}^{\dagger}=-\langle\pi| .
$$

Employing our isospin conventions for the nucleon states: summarized in Appendix B 1 we conclude that:

$$
\mathcal{G}\left|N_{p}\right\rangle=\eta_{N}^{*}(-1)\left|\bar{N}^{\bar{n}}\right\rangle ; \quad \mathcal{G}\left|N_{n}\right\rangle=\eta_{N}^{*}\left|\bar{N}^{\bar{p}}\right\rangle
$$

For antinucleon to pion TDAs we can write down the following parametrization

$$
\begin{aligned}
& 4\left\langle\pi_{a}\left|\hat{\bar{O}}_{\alpha \beta \gamma \rho \tau \chi}(1,2,3)\right| \bar{N}^{\iota}\right\rangle \\
& =\left(f_{a}\right)_{\{\alpha \beta \gamma\}}^{\iota} M_{\rho \tau \chi}^{(\pi \bar{N})_{3 / 2}}(1,2,3)+\varepsilon_{\alpha \beta}\left(\sigma_{a}\right)_{\gamma}^{\iota} M_{\rho \tau \chi}^{(\pi \bar{N})_{1 / 2}\{13\}}(1,2,3) \\
& +\varepsilon_{\alpha \gamma}\left(\sigma_{a}\right)^{\iota}{ }_{\beta} M_{\rho \tau \chi}^{(\pi \bar{N})_{1 / 2}\{12\}}(1,2,3),
\end{aligned}
$$

where the tensor $\left(\bar{f}_{a}\right)_{\{\alpha \beta \gamma\}}$, which is totally symmetric in $\alpha, \beta, \gamma$, reads:

$$
\begin{aligned}
& \left(\bar{f}_{a}\right)_{\{\alpha \beta \gamma\}}^{\iota}=\frac{1}{3}\left(\left(\sigma_{a}^{T}\right)_{\alpha}{ }_{\alpha}{ } \varepsilon_{\delta \beta} \delta^{\iota}{ }_{\gamma}+\left(\sigma_{a}^{T}\right)_{\alpha}{ }_{\alpha} \varepsilon_{\delta \gamma} \delta_{\beta}^{\iota}+\left(\sigma_{a}^{T}\right)_{\beta}^{\delta} \varepsilon_{\delta \gamma} \delta^{\iota}{ }_{\alpha}\right) ; \\
& \text { since }\left(\sigma_{a}^{T}\right)_{\alpha}^{\delta}=\left(\sigma_{a}\right)_{\alpha}{ }^{\delta} \equiv \varepsilon_{\alpha \kappa}\left(\sigma_{a}\right)^{\kappa}{ }_{\theta} \varepsilon^{\theta \delta}
\end{aligned}
$$

The isospin and permutation symmetry identities for the isospin- $\frac{1}{2}$ and isospin- $\frac{3}{2}$ invariant $\pi \bar{N}$ TDAs $M^{(\pi \bar{N})_{1 / 2}\{12\}}, M^{(\pi \bar{N})_{1 / 2}\{13\}}$ and $M^{(\pi \bar{N})_{3 / 2}}$ are the same as those for the relevant $\pi N$ TDAs (see Sec. 4 of Ref. [15]). In particular,

$$
M_{\rho \tau \chi}^{(\pi \bar{N})_{1 / 2}\{13\}}(1,2,3)=M_{\rho \chi \tau}^{(\pi \bar{N})_{1 / 2}\{12\}}(1,3,2) .
$$


Now, using $G$-parity operator $\mathcal{G}$ instead of $\mathcal{C}$, we repeat the derivation of App. B2 and establish the link between $\pi N$ and $\pi \bar{N}$ TDAs.

For example,

$$
\begin{aligned}
& -\frac{\sqrt{2}}{3} M^{(\pi N)_{3 / 2}}(1,2,3)+\sqrt{2} M^{(\pi N)_{1 / 2}}(1,2,3) \\
& =\left\langle\pi^{-}\left|\mathcal{G}^{\dagger} \mathcal{G} u_{\rho}(1) \mathcal{G}^{\dagger} \mathcal{G} u_{\tau}(2) \mathcal{G}^{\dagger} \mathcal{G} d_{\chi}(3) \mathcal{G}^{\dagger} \mathcal{G}\right| N_{n}\right\rangle \\
& =(-1) \eta_{q}^{3} \eta_{N}^{*}(C)_{\rho \rho^{\prime}}(C)_{\tau \tau^{\prime}}(C)_{\chi \chi^{\prime}}\left\langle\pi^{-}\left|\left(\bar{d}_{\rho^{\prime}}\right)^{T}(1)\left(\bar{d}_{\tau^{\prime}}\right)^{T}(2)\left(\bar{u}_{\chi^{\prime}}\right)^{T}(3)\right| \bar{N}^{\bar{p}}\right\rangle \\
& =\eta_{q}^{3} \eta_{N}^{*}(C)_{\rho \rho^{\prime}}(C)_{\tau \tau^{\prime}}(C)_{\chi \chi^{\prime}} \\
& \times\left\{-\frac{\sqrt{2}}{3}\left(M_{\rho^{\prime} \tau^{\prime} \chi^{\prime}}^{\left(\pi \overline{x^{\prime}}\right.}(1,2,3)\right)^{T}+\sqrt{2}\left(M_{\rho^{\prime} \tau^{\prime} \chi^{\prime}}^{\left(\pi \overline{n_{1}}\right.}(1,2,3)\right)^{T}\right\} .
\end{aligned}
$$

One may check that the general parametrization for $\pi \bar{N}$ TDAs (B25) is consistent with that for $\pi N$ TDAs (see eq. (54) of Ref. [15]) once

$$
\begin{aligned}
& \left(M_{\rho \tau \chi}^{(\pi \bar{N})_{1 / 2}}(1,2,3)\right)^{T}=\frac{1}{\eta_{N}^{*} \eta_{q}^{3}}\left(C^{\dagger}\right)_{\rho \rho^{\prime}}\left(C^{\dagger}\right)_{\tau \tau^{\prime}}\left(C^{\dagger}\right)_{\chi \chi^{\prime}} M_{\rho^{\prime} \tau^{\prime} \chi^{\prime}}^{(\pi N)_{1 / 2}}(1,2,3) \\
& \left(M_{\rho \tau \chi}^{(\pi \bar{N})_{3 / 2}}(1,2,3)\right)^{T}=\frac{1}{\eta_{N}^{*} \eta_{q}^{3}}\left(C^{\dagger}\right)_{\rho \rho^{\prime}}\left(C^{\dagger}\right)_{\tau \tau^{\prime}}\left(C^{\dagger}\right)_{\chi \chi^{\prime}} M_{\rho^{\prime} \tau^{\prime} \chi^{\prime}}^{(\pi N)_{3 / 2}}(1,2,3)
\end{aligned}
$$

where the transposition refers to the Dirac indices. The Dirac structures $s^{\pi \bar{N}} \equiv$ $\left\{v_{1,2}^{\pi \bar{N}}, a_{1,2}^{\pi \bar{N}}, t_{1,2,3,4}^{\pi \bar{N}}\right\}$ occurring in the parametrization of the isospin- $\frac{1}{2}$ and isospin- $\frac{3}{2}$ invariant amplitudes $M^{(\pi \bar{N})_{1 / 2}}$ and $M^{(\pi \bar{N})_{3 / 2}}$ are defined by

$$
\left(s_{\rho \tau, \chi}^{\pi \bar{N}}\right)^{T}=\left(C^{\dagger}\right)_{\rho \rho^{\prime}}\left(C^{\dagger}\right)_{\tau \tau^{\prime}}\left(C^{\dagger}\right)_{\chi \chi^{\prime}} s_{\rho^{\prime} \tau^{\prime}, \chi^{\prime}}^{\pi N}
$$

where $s_{\rho^{\prime} \tau^{\prime}, \chi^{\prime}}^{\pi N}$ are the Dirac structures occurring in the parametrization of $\pi N$ TDAs (see eq. (12) of Ref. [15]). For the relevant $\pi \bar{N}$ TDAs (3) we, thus, get

$$
H^{\pi \bar{N}}\left(x_{1}, x_{2}, x_{3}, \xi, \Delta^{2}\right)=\frac{1}{\eta_{N}^{*} \eta_{q}^{3}} H^{\pi N}\left(x_{1}, x_{2}, x_{3}, \xi, \Delta^{2}\right)
$$

\section{AMPLITUDE CALCULATION WITHIN THE $u$-CHANNEL FACTORIZA- TION MECHANISM}

Below we argue that the amplitude of (1) within the $t$-channel factorization mechanism can be obtained from that within the $u$-channel factorization mechanism (see Sec. 2) with 
the obvious change of the kinematical variables:

$$
\begin{aligned}
& p_{N} \rightarrow p_{\bar{N}} ; \quad p_{\bar{N}} \rightarrow p_{N} \\
& \Delta^{u} \rightarrow \Delta^{t} \quad(u \rightarrow t) \\
& \xi^{u} \rightarrow \xi^{t}
\end{aligned}
$$

Since isotopic invariance plays no particular role in the present consideration we consider one flavor of quarks and one "pion" with negative $C$-parity: $\eta_{\pi}=-1$. Generalization for the physical case is straightforward with the use of the $G$-parity formalism described in App. B3.

We introduce the following parametrization for the corresponding matrix elements:

$$
\begin{aligned}
& \left\langle\pi\left|\Psi_{\rho}\left(x_{1}\right) \Psi_{\tau}\left(x_{2}\right) \Psi_{\chi}\left(x_{3}\right)\right| N\left(p_{N}\right)\right\rangle=A_{\rho \tau}\left(p_{N}, \Delta^{u}\right)\left(B U\left(p_{N}\right)\right)_{\chi} H\left(x_{i}, \xi^{u}, u\right) \\
& \left\langle 0\left|\bar{\Psi}_{\bar{\rho}}^{T}\left(y_{1}\right) \bar{\Psi}_{\bar{\tau}}^{T}\left(y_{2}\right) \bar{\Psi}_{\bar{\chi}}^{T}\left(y_{3}\right)\right| \bar{N}\left(p_{\bar{N}}\right)\right\rangle=D_{\bar{\rho} \bar{\tau}}\left(p_{\bar{N}}\right)\left(\bar{V}\left(p_{\bar{N}}\right) E\right)_{\bar{\chi}} \Phi\left(y_{i}\right)
\end{aligned}
$$

where $A\left(p_{N}, \Delta_{u}\right), B, D\left(p_{\bar{N}}\right)$ and $E$ are the relevant Dirac structures; $H\left(x_{i}, \xi^{u}, u\right)$ and $\Phi\left(y_{i}\right)$ denote the scalar TDAs and DAs respectively.

We now express the matrix elements occurring in the $t$-channel factorization scheme through the Dirac structures of the $u$-channel factorization scheme occurring in (C2):

$$
\begin{aligned}
& \left\langle\pi\left|\bar{\Psi}_{\rho}^{T}\left(x_{1}\right) \bar{\Psi}_{\tau}^{T}\left(x_{2}\right) \bar{\Psi}_{\chi}^{T}\left(x_{3}\right)\right| \bar{N}\left(p_{\bar{N}}\right)\right\rangle \\
& =\frac{1}{\eta_{N}^{*} \eta_{q}^{3} \eta_{\pi}} C_{\rho \rho^{\prime}}^{\dagger} C_{\tau \tau^{\prime}}^{\dagger} C_{\chi \chi^{\prime}}^{\dagger}\left\langle\pi\left|\Psi_{\rho^{\prime}}\left(x_{1}\right) \Psi_{\tau^{\prime}}\left(x_{2}\right) \Psi_{\chi^{\prime}}\left(x_{3}\right)\right| N\left(p_{\bar{N}}\right)\right\rangle \\
& =\frac{1}{\eta_{N}^{*} \eta_{q}^{3} \eta_{\pi}}\left(C^{\dagger} A C^{\dagger^{T}}\right)_{\rho \tau}\left(C^{\dagger} B U\right)_{\chi} H\left(x_{i}, \xi^{t}, t\right) ; \\
& \left\langle 0\left|\Psi_{\bar{\rho}}\left(y_{1}\right) \Psi_{\bar{\tau}}\left(y_{2}\right) \Psi_{\bar{\chi}}\left(y_{3}\right)\right| N\left(p_{N}\right)\right\rangle \\
& =\frac{1}{\eta_{N} \eta_{q}^{* 3}} C_{\bar{\rho} \bar{\rho}^{\prime}} C_{\bar{\tau} \bar{\tau}^{\prime}} C_{\bar{\chi} \bar{\chi}^{\prime}}\left\langle 0\left|\bar{\Psi}_{\bar{\rho}^{\prime}}^{T}\left(y_{1}\right) \bar{\Psi}_{\bar{\tau}^{\prime}}^{T}\left(y_{2}\right) \bar{\Psi}_{\bar{\chi}^{\prime}}^{T}\left(y_{2}\right)\right| \bar{N}\left(p_{N}\right)\right\rangle \\
& =\frac{1}{\eta_{N} \eta_{q}^{* 3}}\left(C D C^{T}\right)_{\bar{\rho} \bar{\tau}}\left(\bar{V} E C^{T}\right)_{\bar{\chi}} \Phi\left(y_{i}\right) .
\end{aligned}
$$

In this way we express antinucleon to pion TDAs (respectively, nucleon DAs) through nucleon to pion TDAs (respectively, antinucleon DAs).

Now we proceed with the generic contributions to the amplitude. Each of three lines of the $u$-channel amplitude graph depicted in Fig. 10 corresponds to a matrix in the Dirac 


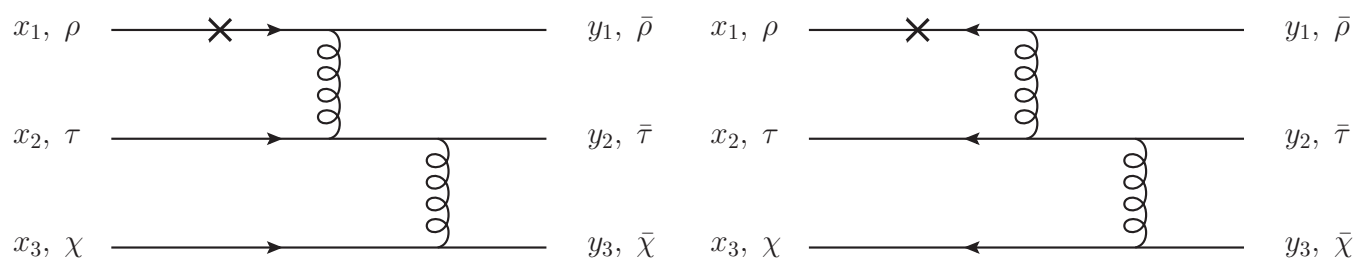

FIG. 10: Typical graphs corresponding to $N \bar{N} \rightarrow \gamma^{*}(q) M\left(p_{M}\right)$. Left panel: $u$-channel regime. Right panel: $t$-channel regime. The crosses represent the virtual photon vertex.

space. We denote these matrices as $\left(L_{1}\right)_{\bar{\rho} \rho},\left(L_{2}\right)_{\bar{\tau} \tau}$ and $\left(L_{3}\right)_{\bar{\chi} \chi}$. Up to the obvious changes due to $p_{N} \leftrightarrow p_{\bar{N}}$, the matrices corresponding to three lines of the $t$-channel graph are $\left(L_{1}^{T}\right)_{\bar{\rho} \rho},\left(L_{2}^{T}\right)_{\bar{\tau} \tau}$ and $\left(L_{3}^{T}\right)_{\bar{\chi} \chi}$.

With these notations the spinor structure of the amplitude corresponding to the $u$ channel graph reads

$$
S^{u}=D_{\bar{\rho} \bar{\tau}}\left(p_{\bar{N}}\right)\left(\bar{V}\left(p_{\bar{N}}\right) E\right)_{\bar{\chi}}\left(L_{1}\right)_{\bar{\rho} \rho}\left(L_{2}\right)_{\bar{\tau} \tau}\left(L_{3}\right)_{\bar{\chi} \chi} A_{\rho \tau}\left(p_{N}, \Delta^{u}\right)\left(B U\left(p_{N}\right)\right)_{\chi} .
$$

The spinor structure of the amplitude corresponding to the $t$-channel graph then reads:

$$
\begin{aligned}
& S^{t} \\
& =\frac{(-1)^{3}}{\eta_{\pi}} D_{\rho \tau}\left(p_{N}\right)\left(\bar{V}\left(p_{N}\right) E\right)_{\chi}\left(C^{\dagger} L_{1}^{T} C\right)_{\rho \bar{\rho}}\left(C^{\dagger} L_{2}^{T} C\right)_{\tau \bar{\tau}}\left(C^{\dagger} L_{3}^{T} C\right)_{\chi \bar{\chi}} A_{\bar{\rho} \bar{\tau}}\left(p_{\bar{N}}, \Delta^{u}\right)\left(B U\left(p_{\bar{N}}\right)\right)_{\bar{\chi}} .
\end{aligned}
$$

Given that for any of 21 relevant graphs presented in Table I of Ref. [13] $L_{1}, L_{2}$ and $L_{3}$ contain an odd number of the Dirac matrices $\gamma_{\mu}$ (and do not contain $\gamma_{5}$ ) we conclude that $S^{u}$ turns into $\eta_{\pi}^{-1} S^{t}$ with the change (C1). Thus we arrive at the conclusion that the $C$ invariance results in a symmetry between the $u$ - and $t$-channel factorization mechanisms. The amplitudes for $N \bar{N} \rightarrow \gamma^{*} \pi$ within the two mechanisms have the forms,

$$
\begin{aligned}
& \mathcal{M}_{s_{N} s_{\bar{N}}}^{\lambda}=\mathcal{C} \frac{1}{Q^{4}}\left[\bar{V}\left(p_{\bar{N}}, s_{\bar{N}}\right) \hat{\epsilon}^{*}(\lambda) \gamma_{5} U\left(p_{N}, s_{N}\right) \mathcal{I}\left(\xi^{u}, u\right)\right. \\
& \left.-\frac{1}{M} \bar{V}\left(p_{\bar{N}}, s_{\bar{N}}\right) \hat{\epsilon}^{*}(\lambda) \hat{\Delta}_{T}^{u} \gamma_{5} U\left(p_{N}, s_{N}\right) \mathcal{I}^{\prime}\left(\xi^{u}, u\right)\right] \\
& \mathcal{M}_{s_{N} s_{\bar{N}}}^{\lambda}=\frac{1}{\eta_{\pi}} \mathcal{C} \frac{1}{Q^{4}}\left[\bar{V}\left(p_{N}, s_{N}\right) \hat{\epsilon}^{*}(\lambda) \gamma_{5} U\left(p_{\bar{N}}, s_{\bar{N}}\right) \mathcal{I}\left(\xi^{t}, t\right)\right. \\
& \left.-\frac{1}{M} \bar{V}\left(p_{N}, s_{N}\right) \hat{\epsilon}^{*}(\lambda) \hat{\Delta}_{T}^{t} \gamma_{5} U\left(p_{\bar{N}}, s_{\bar{N}}\right) \mathcal{I}^{\prime}\left(\xi^{t}, t\right)\right]
\end{aligned}
$$


with the same functions $\mathcal{I}$ and $\mathcal{I}^{\prime}$ (15) standing both in (C8) and (C7). Although a somewhat unusual choice of the Dirac spinors is employed for $S^{t}$ in (C7), this does not lead to any particular complications for the calculation of unpolarized cross sections. Indeed, (C1) can be used at the cross section level after the summation over nucleons spins is performed. As a result, the forward and the backward peaks of the unpolarized cross section possess the identical structure.

[1] M. F. M. Lutz et al. (PANDA Collaboration), arXiv:0903.3905 [hep-ex].

[2] U. Wiedner, Prog. Part. Nucl. Phys. 66, 477 (2011) arXiv:1104.3961 [hep-ex]].

[3] B. Pire and L. Szymanowski, Phys. Lett. B 622, 83 (2005) [arXiv:hep-ph/0504255].

[4] J. P. Lansberg, B. Pire and L. Szymanowski, Phys. Rev. D 76, 111502 (2007) arXiv:0710.1267 [hep-ph]].

[5] J. P. Lansberg, B. Pire, K. Semenov-Tian-Shansky and L. Szymanowski, Phys. Rev. D 85, 054021 (2012) arXiv:1112.3570 [hep-ph]].

[6] L. L. Frankfurt, P. V. Pobylitsa, M. V. Polyakov and M. Strikman, Phys. Rev. D 60, 014010 (1999) arXiv:hep-ph/9901429.

[7] L. Frankfurt, M. V. Polyakov, M. Strikman, D. Zhalov and M. Zhalov, arXiv:hep-ph/0211263.

[8] A. V. Radyushkin, arXiv:hep-ph/0410276.

[9] A. V. Efremov and A. V. Radyushkin, Theor. Math. Phys. 42, 97 (1980) [Teor. Mat. Fiz. 42, $147(1980)]$.

[10] G. P. Lepage and S. J. Brodsky, Phys. Rev. D 22, 2157 (1980).

[11] V. L. Chernyak and A. R. Zhitnitsky, Phys. Rept. 112, 173 (1984).

[12] V.L. Chernyak and I.R. Zhitnitsky, Nucl. Phys. B 246, 52 (1984).

[13] J. P. Lansberg, B. Pire and L. Szymanowski, Phys. Rev. D 75, 074004 (2007) [Erratum-ibid. D 77, 019902 (2008)] hep-ph/0701125.

[14] B. Pire, K. Semenov-Tian-Shansky and L. Szymanowski, Phys. Rev. D 82, 094030 (2010) arXiv:1008.0721 [hep-ph]].

[15] B. Pire, K. Semenov-Tian-Shansky, L. Szymanowski, Phys. Rev. D 84, 074014 (2011) 
arXiv:1106.1851 [hep-ph]].

[16] B. Pasquini, M. Pincetti and S. Boffi, Phys. Rev. D 80, 014017 (2009) arXiv:0905.4018 [hep-ph]].

[17] S. Aoki et al. (JLQCD Collaboration), Phys. Rev. D62, 014506 (2000). hep-lat/9911026.

[18] Y. Aoki, C. Dawson, J. Noaki, A. Soni, Phys. Rev. D75, 014507 (2007). hep-lat/0607002.

[19] M. Burkardt, Phys. Rev. D 62, 071503 (2000) arXiv:hep-ph/0005108].

[20] M. Burkardt, Phys. Rev. D 66, 114005 (2002) arXiv:hep-ph/0209179].

[21] M. Diehl, Eur. Phys. J. C 25, 223 (2002) arXiv:hep-ph/0205208.

[22] J. P. Ralston and B. Pire, Phys. Rev. D 66, 111501 (2002) hep-ph/0110075.

[23] V. I. Borodulin, R. N. Rogalev and S. R. Slabospitsky, hep-ph/9507456.

[24] N. G. Stefanis, Eur. Phys. J. direct C 1, 1 (2000).

[25] V. M. Braun, A. Lenz and M. Wittmann, Phys. Rev. D 73, 094019 (2006) hep-ph/0604050.

[26] V. L. Chernyak, A. A. Ogloblin and I. R. Zhitnitsky, Z. Phys. C 42, 583 (1989) [Yad. Fiz. 48, 1398 (1988)] [Sov. J. Nucl. Phys. 48, 889 (1988)].

[27] I. D. King and C. T. Sachrajda, Nucl. Phys. B 279, 785 (1987).

[28] A. Lenz, M. Gockeler, T. Kaltenbrunner and N. Warkentin, Phys. Rev. D 79, 093007 (2009) arXiv:0903.1723 [hep-ph]].

[29] J. Bolz and P. Kroll, Z. Phys. A 356, 327 (1996) hep-ph/9603289].

[30] O. Dumbrajs, R. Koch, H. Pilkuhn, G. c. Oades, H. Behrens, J. j. De Swart and P. Kroll, Nucl. Phys. B 216, 277 (1983).

[31] D. Mueller, B. Pire, L. Szymanowski and J. Wagner, Phys. Rev. D 86, 031502 (2012) arXiv:1203.4392 [hep-ph]].

[32] C. Adamuscin, E. A. Kuraev, E. Tomasi-Gustafsson and F. E. Maas, Phys. Rev. C 75, 045205 (2007) hep-ph/0610429.

[33] J. Guttmann and M. Vanderhaeghen, arXiv:1210.3290 [hep-ph].

[34] C. Itzykson, J. B. Zuber, Quantum Field Theory, (McGraw-Hill, New York, USA 1980).

[35] T. Ericson, W. Weise, Pions and Nuclei, (Clarendon Press, Oxford, England 1988). 